\title{
A Theory of Large Fluctuations in Stock Market Activity*
}

\author{
Xavier Gabaix ${ }^{\dagger}$, Parameswaran Gopikrishnan ${ }^{\ddagger}$, Vasiliki Plerou ${ }^{\ddagger}$, H. Eugene Stanley ${ }^{\ddagger}$ \\ ${ }^{\dagger} \mathrm{MIT}$, Economics Department and NBER \\ ${ }^{\ddagger}$ Boston University, Physics Department, Center for Polymer Studies
}

August 16, 2003

\begin{abstract}
We propose a theory of large movements in stock market activity. Our theory is motivated by growing empirical evidence on the power-law tailed nature of distributions that characterize large movements of distinct variables describing stock market activity such as returns, volumes, number of trades, and order flow. Remarkably, the exponents that characterize these power laws are similar for different countries, for different types and sizes of markets, and for different market trends, suggesting that a generic theoretical basis may underlie these regularities. Our theory provides a unified way to understand the power-law tailed distributions of these variables, their apparently universal nature, and the precise values of exponents. It links large movements in market activity to the power-law distribution of the size of large financial institutions. The trades made by large financial institutions create large fluctuations in volume and returns. We show that optimal trading by such large institutions generate power-law tailed distributions for market variables with exponents that agree with those found in empirical data. Furthermore, our model also makes a large number of testable out-of-sample predictions.
\end{abstract}

\section{Contents}

1 Introduction $\quad \mathbf{4}$

1.1 The power-law distribution of returns, volume, number of trades . . . . . . . . . 4

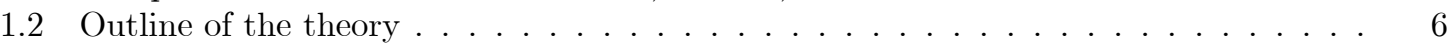

2 The empirical facts that motivate our theory $\quad 7$

2.1 Methodology to estimate power laws . . . . . . . . . . . . . . . . . . 7

2.2 Normalizations used to make different assets comparable . . . . . . . . . . . . . . 7

2.3 The cubic law of price fluctuations: $\zeta_{r} \simeq 3 \ldots \ldots . \ldots$. . . . . . . . . . 8

*xgabaix@mit.edu, gopi@cgl.bu.edu, plerou@cgl.bu.edu, hes@miranda.bu.edu. We thank Tal Fishman, David Kang, Alex Weisgerber, and especially Kirk Doran for outstanding research assistance. For helpful comments, we thank Tobias Adrian, Jonathan Berk, Olivier Blanchard, Jean-Phillipe Bouchaud, John Campbell, Emanuel Derman, Ken French, Joel Hasbrouck, Soeren Hvidjkaer, Harrison Hong, Ivana Komunjer, David Laibson, Augustin Landier, Ananth Madhavan, Lasse Pedersen, Thomas Philippon, Marc Potters, Jon Reuter, Gideon Saar, Andrei Shleifer, Didier Sornette, Dimitri Vayanos, Jessica Wachter, Jiang Wang, Jeff Wurgler, and seminar participants at Berkeley's Haas School, Delta, Harvard, John Hopkins University, MIT, NBER, NYU's Stern School, Princeton, Stanford GSB, the 2002 Econophysics conferences in Indonesia and Tokyo, and the 2003 WFA meetings. We thank the NSF for support. X.G. thanks the Russell Sage Foundation and NYU Stern School for their wondeful hospitality during the year 2002-2003. 
2.4 The half cubic law of volume: $\zeta_{Q} \simeq 3 / 2 \ldots \ldots \ldots \ldots$

2.5 The cubic law of number of trades: $\zeta_{N} \simeq 3.3 \ldots \ldots \ldots \ldots \ldots \ldots$

2.6 The power law distribution of the size of large investors: $\zeta_{S} \simeq 1 \ldots \ldots$

3 The theory, assuming a power law price impact of trades 11

3.1 Sketch of the theory . . . . . . . . . . . . . . . . . . . . 11

3.2 Notations and some elementary links between exponents . . . . . . . . . . . . . . 12

3.2 .1 Notations . . . . . . . . . . . . . . . . . . . . . 12

3.2 .2 Link between aggregate and trader-based exponents . . . . . . . . . . . 13

3.2.3 Link between the exponents of return and volume . . . . . . . . . . . . 13

3.3 A main result . . . . . . . . . . . . . . . . . . . . . . . . . . . . . 14

3.4 An optimizing model that illustrates Theorem $3 \ldots \ldots \ldots$

4 The square root of price impact of trades: Evidence and a possible explanation 19

4.1 Evidence on the square root law of price impact . . . . . . . . . . . . . . . . 19

4.2 Sketch of our explanation for the square root price impact . . . . . . . . . . . . 19

4.3 A microstructure model for the power law impact of a block . . . . . . . . . . . . . 21

4.3.1 The behavior of liquidity providers . . . . . . . . . . . . . . . 21

4.3.2 Permanent vs Transitory price impact . . . . . . . . . . . . . . . . . . 21

4.3.3 The behavior of the large trader . . . . . . . . . . . . . . . 21

4.4 The distribution of individual trades $q$ vs distribution of target volumes $Q \ldots \ldots$

4.5 An optimizing intertemporal model that joins power laws and microstructure . . . . 24

4.6 Some comments on the model . . . . . . . . . . . . . . . . . . . . 25

$4.6 .1 \quad$ Arbitrage . . . . . . . . . . . . . . . . . 25

4.6.2 Plausibility of very large volumes and price impact $\ldots \ldots \ldots 26$

5 Assessing some further empirical predictions of the model 26

5.1 Contemporaneous behavior of several measures of trading activity . . . . . . . . . 26

5.1 .1 Definitions . . . . . . . . . . . . . . . . . 26

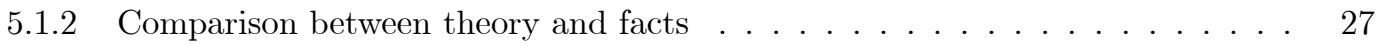

5.2 Some untested predictions . . . . . . . . . . . . . . . . . 28

6 Related literature $\quad 28$

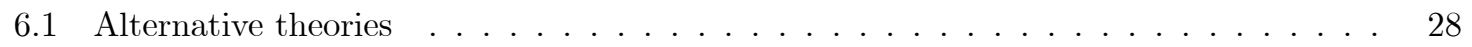

6.1.1 The public news based (efficient markets) model . . . . . . . . . . . . . 30

6.1.2 A mechanical "price reaction to trades" model . . . . . . . . . . . . . . . . . 30

6.1 .3 Random bilateral matching . . . . . . . . . . . . . . . . . . . 30

6.2 Related empirical findings . . . . . . . . . . . . . . . . . . . . . 30

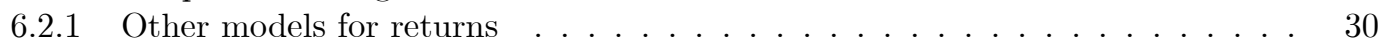

6.2 .2 Buy / Sell asymmetry . . . . . . . . . . . . . . . . . . . . 31

6.3 Link with the behavioral and excess volatility literature $\ldots \ldots \ldots \ldots \ldots$

6.4 Link with the microstructure literature . . . . . . . . . . . . . . . . . . 31

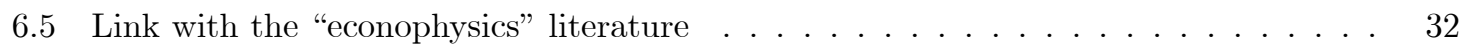

$\begin{array}{lll}7 & \text { Conclusion } & 32\end{array}$

8 Appendix A: Some power law mathematics $\quad 34$

9 Appendix B: Generalization of Theorem 3 to non i.i.d. settings 35 
10 Appendix C: The model with general trading exponents

11 Appendix D: A model for the linear supply function (38)

12 Appendix E: Algorithm for the simulations

13 Appendix F: Confidence intervals and tests when a variable has infinite variance 41 13.1 Construction of the confidence intervals for Figure $7 \ldots \ldots \ldots$. . . . . . . 41

13.2 Test of the linear relation $E\left[r^{2} \mid Q\right]=\alpha+\beta V \ldots \ldots \ldots \ldots \ldots \ldots$ 


\section{Introduction}

Even ex post, stock market fluctuations are very hard to explain by movement in fundamentals. Trading per se seems to move prices. This leads to excess volatility of stock market prices. Even crashes seem to happen for no good reason. Trading volume is very high, and its large variations are also hard to relate to fundamentals. The present paper wishes to present a theory of those large movements in trading activity ${ }^{1}$.

For this is useful to have precise empirical facts. Otherwise, theories are too unconstrained. This is why we use a series of sharp empirical facts, established using dozens of millions of data points. They are quite precise, hence they constrain any theory of large movements of stock market activity. We first outline some of those key empirical regularities before presenting our theory.

\subsection{The power-law distribution of returns, volume, number of trades}

Our theory is motivated by the following empirical findings on the power law distribution of (i) returns, (ii) volumes, and (iii) the number of trades, (iv) the power law of price impact, and (v) the power law distribution of the size of large investors.

(i) The power law distribution of returns. Let $P_{t}$ denote the price of a stock or an index, and define return over a time interval $\Delta t$ as $r_{t}=\ln \left(P_{t} / P_{t-\Delta t}\right)$. Empirical studies (Gopikrishnan et al. (1999), Plerou et al. (1999)) show that the distribution function of returns for 1000 largest U.S. stocks and several major international indices decays as

$$
P(|r|>x) \sim \frac{1}{x^{\zeta_{r}}} \text { with } \zeta_{r} \simeq 3
$$

Here, $\sim$ means asymptotic equality up to numerical constants ${ }^{2}$. This holds for positive and negative returns separately.

Figure 1 shows that $P(|r|>x)$ on a bi-logarithmic scale displays a linear asymptotic behavior with slope $-\zeta_{r}=-3.1$. Since linear behavior on a log-log plot means a power-law decay, we conclude that $\ln P(|r|>x)=-3.1 \ln x+$ constant, i.e. (1). There is no tautology that implies that this graph should be a straight line, or that the slope should be -3. A Gaussian would have a concave parabola, not a straight line. The remarkable nature of the distribution (1) is that it holds for stock indices and individual stocks different sizes, different time periods (see Section 2 for a systematic exploration), and we always find $\zeta_{r} \simeq 3$. In the following, we shall refer to Eq. (1) as "the cubic law of returns".

(ii) The power law distribution of trading volume. We call volume the number of shares traded ${ }^{3}$. We find that the distribution is:

$$
P(q>x) \sim \frac{1}{x^{\zeta_{q}}} \text { with } \zeta_{q} \simeq 3 / 2 .
$$

The finding holds both for the volume of individual trades and aggregate volumes:

$$
P(Q>x) \sim \frac{1}{x^{\zeta_{Q}}} \text { with } \zeta_{Q} \simeq 3 / 2
$$

\footnotetext{
${ }^{1}$ A condensed version of some elements of the present paper appeared in Gabaix et al. (2003a).

${ }^{2}$ Formally $f(x) \sim g(x)$ means $f(x) / g(x)$ tends to a positive constant (not necessarily 1 ) as $x \rightarrow \infty$.

${ }^{3}$ The dollar value traded yields very similar results. This is expected, as, for a given security, the variations of number of shares traded and the volume traded are essentially proportional.
} 


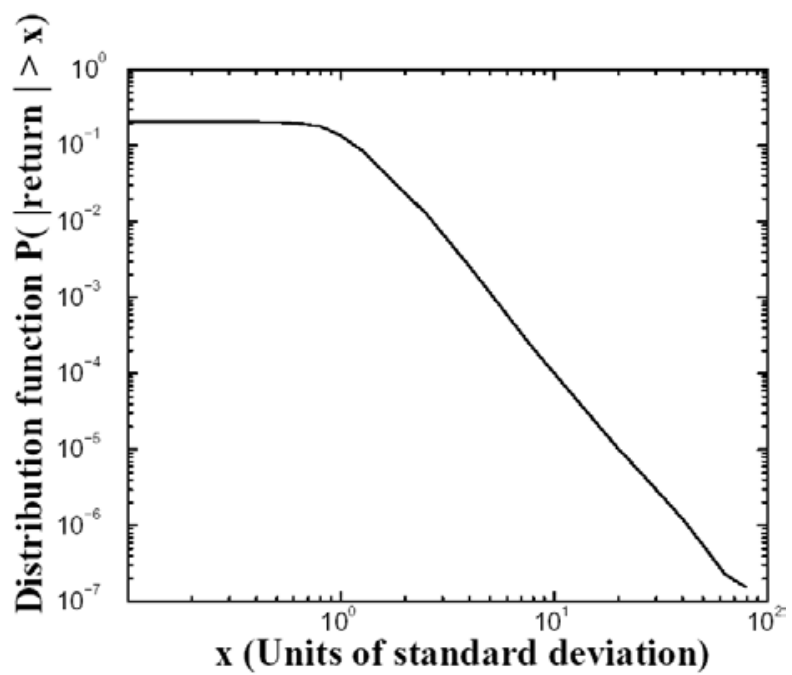

Figure 1: Empirical cumulative distribution of the absolute values of the normalized 15 minute returns of the 1000 largest companies in the TAQ database for the 2-yr period 1994-1995. This represents 12 million observations. In the region $2<x<80$ we find an OLS fit $\ln P(r>x)=$ $-\zeta_{r} \ln x+b$, with $\zeta_{r}=3.1 \pm 0.1$. This means that returns are distributed with a power law $P(r>x) \sim$ $x^{-\zeta_{r}}$ for large $x$.

We had initially found this in U.S. data (Gopikrishnan et al. 2000), and we have recently confirmed the same on French data (Plerou et al. 2003). Figure 2 illustrates this. It shows the density satisfies $p(q) \sim q^{-2.5}$, i.e. (2). The exponent of the distribution of individual trades is close to 1.5. In the following, we refer to Eq. (2)-(3) as the "half-cubic law of trading volume".

As before, the exponents describing these power-laws seem to be stable across different types of stocks, different time periods and time horizons etc. (see Section 2).

(iii) The power law distribution of the number of trades. A similar analysis for the number of trades shows that

$$
P(N>x) \sim \frac{1}{x^{\zeta_{N}}} \text { with } \zeta_{N} \simeq 3.3
$$

(iii) The power law of price impact.

Building on the concave nature of the impact functions in stock markets (Hasbrouck 1991, Hasbrouck and Seppi 2001, Plerou et al. 2002), we will give evidence that the price impact $\Delta p$ of a trade of size $V$ scales as:

$$
\Delta p \sim V^{\gamma} \text { with } \gamma \simeq 1 / 2 .
$$

(v) Power law distribution of the assets of large investors. Our empirical analysis of the size distribution of mutual funds shows evidence for a power law distribution. As Section 2.6 reports the number of funds with size (asset under management) greater than $x$ follows:

$$
P(S>x) \sim \frac{1}{x^{\zeta_{S}}} \text { with } \zeta_{S} \simeq 1
$$

We will present a theory where those five facts are intimately related. It is largely orthogonal to the existing finance theory. Indeed, much of finance is about the risk premia commanded by various 


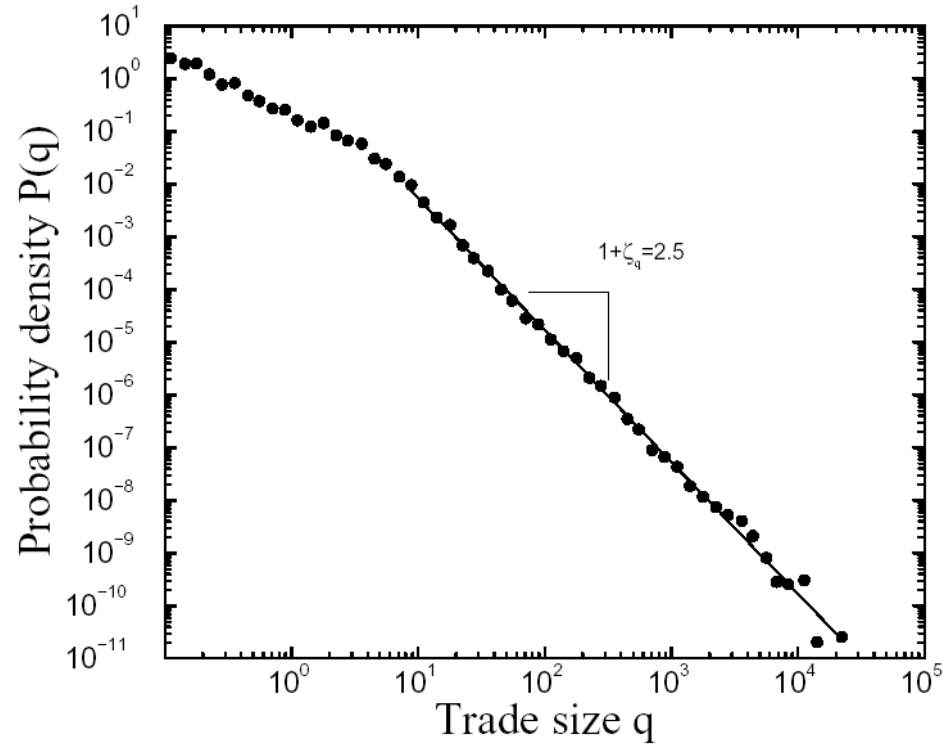

Figure 2: Probability density of the individual trade sizes $q$ for 30 large stocks in the Paris Bourse from January 1995 to October 1999. This represents 35 million observations. OLS fit yields a density $\sim q^{-\left(1+\zeta_{q}\right)}$, for $\zeta_{q}=1.5 \pm 0.1$. Source: Plerou et al. (2003).

shocks, but not about the origins of the shocks themselves. For instance, if $r_{M t}$ is a market return, and $\varepsilon_{i t}$ is an idiosyncratic return, a typical finance model explains why an asset $i$ will have a return of the factor type:

$$
r_{i t}=\beta_{i} r_{M t}+\varepsilon_{i t}
$$

what causes variations in the loadings $\beta_{i}$, or even what the relevant factors $r_{M}$ should be. In contrast, our goal here is not to explain risk premia, but rather to propose a structured theory for the origin of the shocks $r_{M t}, \varepsilon_{i t}$. For this we are guided by the regularities in their distribution and in the way they relate to other market quantities such as volume and volume "imbalance". In other words, we wish to theorize on the origins of those movements, especially to the extent that they cannot easily be related to fundamentals.

\subsection{Outline of the theory}

In our theory, large volumes and large returns are created by the trades of large investors. To see this qualitatively, suppose that a large investor decides to trade in a stock due to some news announcement, or some recent analysis he made himself. For a sufficiently large investor, the desired quantity of the particular stock will be comparable to the daily turnover ${ }^{4}$. So if he executes the trade quickly he will suffer a large price impact. However, the alternative of performing the trade very slowly over time is not attractive either since the mispricing cannot be expected to remain indefinitely, so he does not wish to wait too long before realizing the trade. We show that under plausible conditions, his "optimal" trading behavior generates power law exponents for returns,

\footnotetext{
${ }^{4}$ Please see Section 4.6.2 for empirical support of this assertion.
} 
volumes and number of trades, i.e. the exponents $\zeta_{Q}=\zeta_{q}=3 / 2$ for the volume, and $\zeta_{r}=\zeta_{N}=3$ for the return and number of trades.

Section 2 presents the data and our empirical findings in more detail. The provide a self-contained tour of the empirical literature on power laws. For modularity's sake, we chose to present the model in two steps. In Section 3, we present the model while assuming the microstructure part, i.e. assuming a power law price impact. We show how his generate the above power laws ${ }^{5}$. In Section 4, we present one possible model for the square root price impact of trade. Section 5 gathers the out-of-sample predictions of our model and our empirical results for some of these predictions. Section 6 discusses our finding in the context of the relevant literatures. Section 7 presents concluding remarks and directions of future work.

\section{The empirical facts that motivate our theory}

\subsection{Methodology to estimate power laws}

There are two basic methodologies to estimate power laws exponents. We illustrate them with the examples of returns. In both methods, one selects a cutoff of returns, and orders the top $n$ observations as $r_{(1)} \geq \ldots \geq r_{(n)}$, up to a certain cutoff.

The simplest method is to do a "log rank log size regression", where an estimate of $\zeta$ is the the OLS coefficient on $r_{(i)}$ in the regression of $\log$ of the rank $i$ (or of cumulative frequency $i / n$ ) on the $\log$ size:

$$
\ln i / n=A-\widehat{\zeta}^{O L S} \ln r_{(i)}+\text { noise }
$$

This method is the simplest, and yields a visual goodness of fit with the power law. This what we do for instance in Figure 1.

A second method is Hill's estimator

$$
\widehat{\zeta}^{\text {Hill }}=\frac{n-1}{\sum_{i=1}^{n-1}\left(\ln r_{(i)}-\ln r_{(n)}\right)} .
$$

Those two methods have pitfalls. In particular, they underestimate the true standard errors. Some of those pitfalls are discussed in Embrechts et al. (1997, 330-345) and Gabaix and Ioannides (2004).

Most of the available statistical literature assumes i.i.d. draws. In contrast, there is a clear autocorrelation of volatility and trading activity in financial data. This does not bias our estimates of the unconditional distributions, but makes the assessment of the standard errors more difficult. Before the statistically rigorous theory of how to proceed is developed, our pragmatic solution is to use a large amount of data - several million observations. The stability of the estimates across different periods, countries and classes of assets give us anyway confidence that the empirical estimates we report here are robust.

\subsection{Normalizations used to make different assets comparable}

To compare quantities across different stocks, we normalize variables such as $r, V$ and $N$ by the second moments in they exist, otherwise by the first moments ${ }^{6}$. For instance, for a stock $i$, we consider the returns $\widehat{r}_{i t}=\left(r_{i t}-r_{i}\right) / \sigma_{r, i}$, where $r_{i}$ is the mean of the $r_{i t}$ and $\sigma_{r, i}$ is their standard

\footnotetext{
${ }^{5}$ Technical extension of the present paper are available in Gabaix et al. (2003b).

${ }^{6}$ This is explained more systematically in Appendix F.
} 


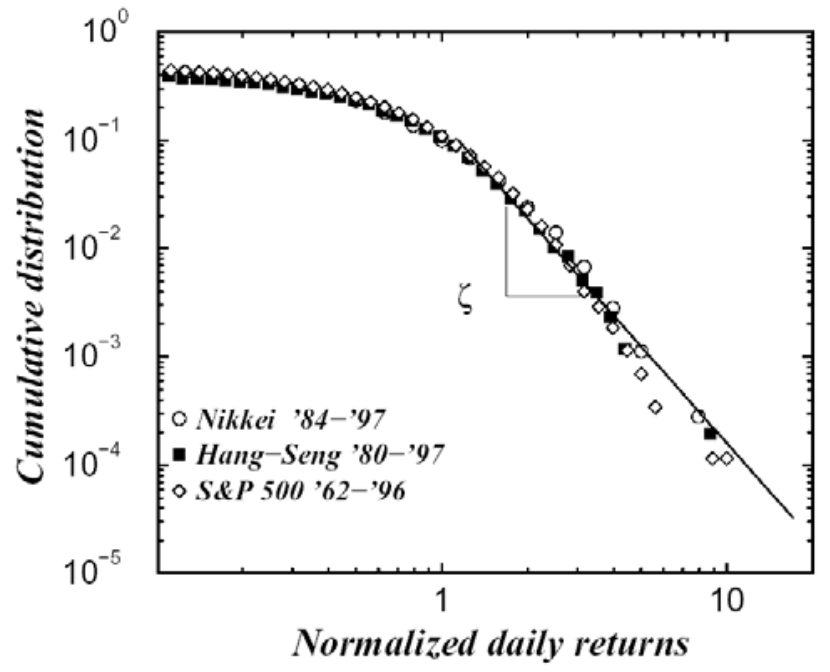

Figure 3: Zipf plot for the daily fluctuations in the Nikkei (1984-97), the Hang-Seng (1980-97), and the S\&P 500 (1962-96). The apparent power-law behavior in the tails is characterized by the exponents $\zeta_{r}=3.05 \pm 0.16$ (NIKKEI), $\zeta_{r}=3.03 \pm 0.16$ (Hang-Seng), and $\zeta_{r}=3.34 \pm 0.12$ (S\&P 500). The fits are performed in the region $|r|>1$. Source: Gopikrishnan et al. (1999).

deviation. For the volume, which has an infinite standard deviation, we consider the normalization: $\widehat{Q}_{i t}=Q_{i t} / Q_{i}$, where $Q_{i}$ is the mean of the $Q_{i t}$.

\subsection{The cubic law of price fluctuations: $\zeta_{r} \simeq 3$}

The cubic distribution of returns has been uncovered in a series of studies (Lux 1996, Gopikrishnan et al. 1999, Plerou et al. 1999). We show here the robustness of the power-law exponent $\zeta_{r} \simeq 3$.

It seems to hold internationally. We analyze data for 10 other developed stock markets ${ }^{7}$. We find a mean of $\zeta_{r}=2.9$ with a standard error of 0.10 . Figure 3, for instance, shows how the return distribution of three different countries are very similar.

The cubic law predicts the existence of a few very large returns over a century. In Gabaix et al. (2003c) we show that in the U.S., stock market crashes, including the 1929 and 1987 crashes, are not outliers to the cubic law. Hence a theory of the cubic law of returns might be a theory of crashes.

Mandelbrot (1963) argued that financial return followed a Lévy stable distribution, and offered the theory that this might come from a large number of shocks with infinite variance. Lévy distribution imply that $\zeta_{r} \leq 2$. But our data are inconsistent with $\zeta_{r} \leq 2$, hence we are lead to reject Mandelbrot's "stable Paretian hypothesis" (Fama 1963).

Having checked the robustness of the $\zeta=3$ finding across different stock markets, we look at firms of different sizes. Small firms have a higher volatility than big firms, as is verified in Figure

\footnotetext{
${ }^{7}$ We took the stock market indices of Australia (1/1/73-11/27/01) (1/2/84-11/17/01), Canada (1/1/69-11/20/01), France (1/2/84-11/27/01), Germany (1/1/73, 10/18/01), Japan (1/1/69-11/30/01), Hong-Kong, $(1 / 1 / 73-11 / 30 / 01)$, Netherlands (1/1/80-11/27/01), South Korea (1/7/75-11/27/01), Spain $(1 / 2 / 74-11 / 27 / 01)$, and United Kingdom $(12 / 25 / 68-11 / 20 / 01)$. We calculated the Hill estimator for the extreme $1 \%$ of the distribution.
} 

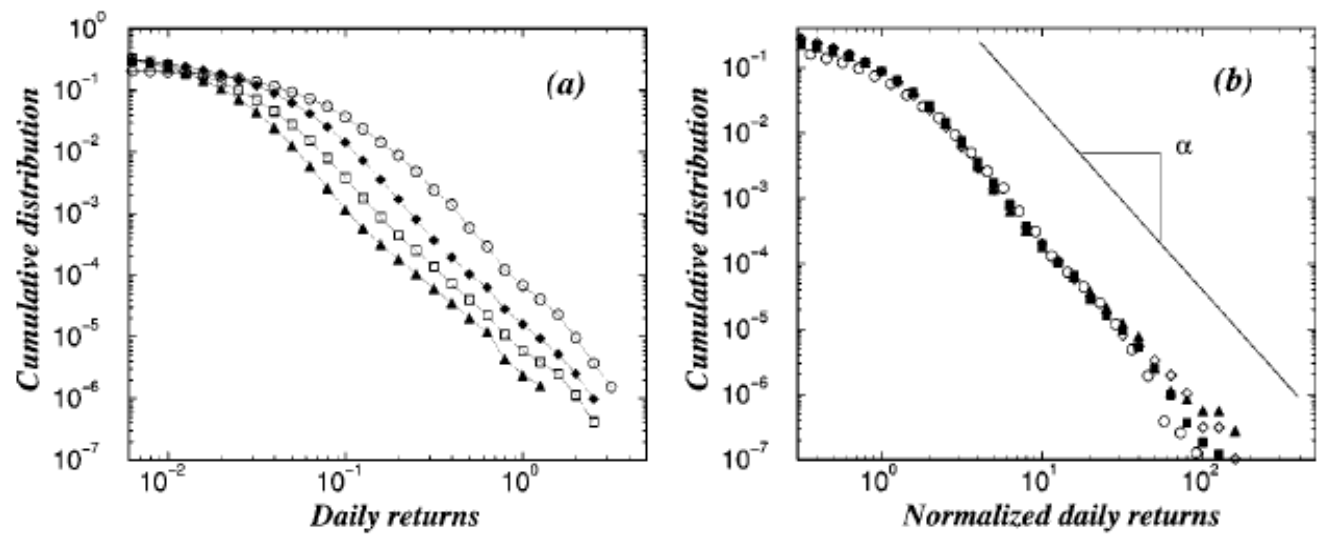

Figure 4: Cumulative distribution of the conditional probability $P(r>x)$ of the daily returns of companies in the CRSP database, 1994-5. We consider the starting values of market capitalization $K$, and define uniformly spaced bins on a logarithmic scale and show the distribution of returns for the bins, $K \in\left(10^{5}, 10^{6}\right], K \in\left(10^{6}, 10^{7}\right], K \in\left(10^{7}, 10^{8}\right], K \in\left(10^{8}, 10^{9}\right]$. (a) Unnormalized returns (b) Returns normalized by the average volatility $\sigma_{K}$ of each bin. The plots collapsed to an identical distribution, with $\zeta_{r}=2.70 \pm .10$ for the negative tail, and $\zeta_{r}=2.96 \pm .09$ for the positive tail. Source: Plerou et al. (1999).

4(a). But the same figure also shows a similar slope. Indeed, when we normalize the distribution by a common standard deviation, we see that the plots collapse, and the exponents are very similar, around $\zeta=3$ again.

Some studies have quantified the power law exponent of foreign exchange fluctuations. The most comprehensive is probably Guillaume et al. (1997), who calculate the exponent $\zeta_{r}$ of the price movements between the major currencies. At the shortest frequency $\Delta t=10$ minutes, they find exponents with average $\left\langle\zeta_{r}\right\rangle=3.44$, and a standard deviation 0.30 . This tantalizingly close to the stock market findings, though the standard error is too high to draw sharp conclusions ${ }^{8}$.

\subsection{The half cubic law of volume: $\zeta_{Q} \simeq 3 / 2$}

Trying to understand the origins of the cubic law for returns, Gopikrishnan et al. (2000a) looked at the distribution of volume ${ }^{9}$ in the U.S. and found an exponent around 1.5: $\zeta_{Q}=1.53 \pm .07$. The same value $\zeta_{q} \simeq 3 / 2$ held for the distribution of individual trades. Maslov and Mills (2001) find likewise $\zeta_{q}=1.4 \pm .1$ for the volume of market orders. To test the universality of this finding, we examine 30 large stocks of the Paris Bourse from 1995-2000, which contains approximately 35 million records. We reported the finding in Figure 2 and found $\zeta_{q}=1.5 \pm .1$.

\footnotetext{
${ }^{8}$ Suppose returns are distributed according to a cubic law with a the density proportional $1 /\left(1+r^{2}\right)^{2}$. Examining the top $1 \%$ of 10,000 points, the standard deviation of measured exponents is about 0.4 , for a mean of about 3 .

${ }^{9}$ For stock $i$, we look at the fluctuations of $V_{i t} / V_{i}$, where $V_{i}$ is the mean volume $V_{i t}$. This makes stocks comparable, and the "number of shares" and "dollar volume" would give equivalent measures of volume.
} 


\subsection{The cubic law of number of trades: $\zeta_{N} \simeq 3.3$}

Plerou et al. (2001) find an exponent $\zeta_{N}=3.4 \pm .05$ for the number of trades in a 15 min interval in the U.S. In the Paris Bourse data mentioned above, we find $\zeta_{N}=3.17 \pm .1$. Our theory will predict the exponent of 3 . This may due to a flaw in the theory ${ }^{10}$, or to simply to measurement error ${ }^{11}$.

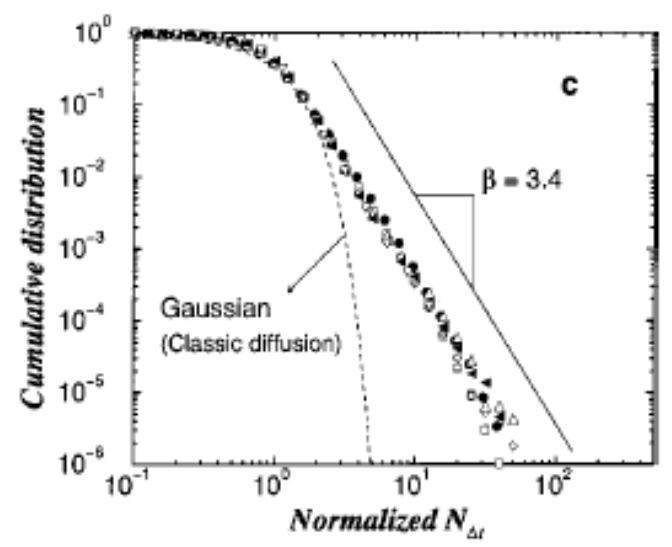

Figure 5: Cumulative distribution of the normalized number of transactions $n_{\Delta t}=N_{\Delta t} /\left\langle N_{\Delta t}\right\rangle$. Each symbol shows the cumulative distribution $P\left(n_{\Delta t}>x\right)$ of the normalized number of transactions $n_{\Delta t}$ for all stocks in each bin of stocks sorted according to size. An analysis of the exponents obtained by fits to the cumulative distributions of each of the 1000 stocks yields an average value $\zeta_{N}=\beta=3.40 \pm 0.05$.

\subsection{The power law distribution of the size of large investors: $\zeta_{S} \simeq 1$}

The size distribution of many entities follows a power law, and often with exponent 1, something called a Zipf's law. This is true for cities (Zipf 1949, Gabaix and Ioannides 2003) and business firms. Indeed Axtell (2001) and Okuyama et al. (1999) have shown that the distribution of firms (respectively in the US and Japan) follows Zipf's law. As they show that the distribution of firms follows Zipf's law, it is tempting to hypothesize that the distribution of firms that manage money follows Zipf's law. We found data for an important subset of those firms, mutual funds ${ }^{12}$. From CRSP one gets the size (dollar value of the assets under management) of all the mutual funds ${ }^{13}$

\footnotetext{
${ }^{10}$ A scaling $N \sim V^{\xi}$ gives a value $\zeta_{N}=\zeta_{V} / \xi$, so that a scaling of the number of trades $N \sim V^{0.44}$ rather that the theoretical $N \sim V^{0.5}$ would correct this misfit. This would mean that the broker can do slightly better than predicted by the model. For very large trade, the broker could have an extra incentive to limit the number of people to whom the trade is offered, perhaps to limit information leakage.

${ }^{11}$ Power law relations are notoriously hard to measure, especially for positive variables with high exponents with positive quantities. The reason is that high exponents have fewer very large events, so the measurement can be affected by other sources of noise. For instance, suppose that the true process for the number of trades $N$ is $N=$ $N_{0}+a$, where $N_{0}$ has power law 3 with $P\left(N_{0}>x\right)=k x^{-3}$, and $a$ is a constant. The interpretation is that $a$ is a constant "background noise" number of trades, and the $N_{0}$ represents the trades of large investors as in our model. Then the CDF of $N$ is $G(x)=P(N>x)=k(x-a)^{-3}$, and the measured power law exponent will be: $\widehat{\zeta_{N}}=-x G^{\prime}(x) / G(x)=/(1-a / x)$. It tends to 3 for very large values of $x$, but in finite samples, there is an upward bias equal to $a / \overline{N_{0}}$, where $\overline{N_{0}}$ is the order of magnitude of typical value of $N_{0}$ in the sample.

${ }^{12}$ Here we sketch the main findings. Gabaix, Ramalho and Reuter (2003) present many more details.

${ }^{13}$ The say 200 funds of Fidelity, for instance, count as 200 different funds, not as one big "Fidelity" fund.
} 
from 1961-1999. For each year $t$, we do an OLS estimation of the power law exponent $\zeta$ of the distribution. We find an average coefficient $\left\langle\zeta_{t}\right\rangle=1.10$, with a standard deviation across years ${ }^{14}$ of 0.08. The Hill estimator technique gives a mean estimate $\left\langle\zeta_{t}\right\rangle=0.90$ and a standard deviation $\left\langle\zeta_{t}^{2}-\left\langle\zeta_{t}\right\rangle^{2}\right\rangle^{1 / 2}=0.07$. All in all we conclude that, to a good approximation, mutual fund sizes follow a power law distribution with exponent:

$$
\zeta_{S} \simeq 1
$$

For the purpose of this paper, one can take this distribution of the sizes of mutual funds as a given. It is in fact not difficult to explain. One can transpose the explanations given for cities (Gabaix 1999, and the references in Gabaix and Ioannides 2004) to mutual funds. A log normal process with small perturbation to ensure convergence to a non-degenerate steady-state distribution, explains the power law distribution with an exponent of 1. Gabaix, Ramalho and Reuter (2003) develops this idea and shows that those assumptions are verified empirically.

It is only recently, say in the past 30 years, that mutual funds have come to represent a large part of the marketplace. For the earlier periods, the theory will work if the distribution of large agents still follow Zipf's law with a unit exponent. We do not have direct evidence for this, but a very natural candidate would be the pension funds of corporations. It is very likely that the size of the pension fund of a firm with $S$ employees is proportional to $S$, so that their pension funds also follow Zipf's law.

\section{The theory, assuming a power law price impact of trades}

\subsection{Sketch of the theory}

Assuming a power-law functional form for price impact, we will show how the power law distribution of large investors generates, through intelligent ${ }^{15}$, tactical behavior of the traders, the exponents $\zeta_{Q}=\zeta_{q}=3 / 2$ for the volume, and $\zeta_{r}=\zeta_{N}=3$ for returns, volumes and number of trades. The origins of the power law distribution of large investors are fairly well understood ${ }^{16}$. In Section 4 , we will provide an explanation for the power law price impact.

In our theory, large volumes and large returns are created by trades made by large agents (investors). When a large agent wants to trade with reasonable speed, he moves the market. Trading slowly decreases the direct price impact, but has other drawbacks, as the profitability of the trading opportunity may diminish in part due to information leakages. We will show that optimal trading by large investors generates the exponents $\zeta_{r}=\zeta_{N}=3$ and $\zeta_{q}=3 / 2$.

Figure 6 displays the modular structure of the paper. It indicates the logical relations of the different parts of our theory. Its different parts are fairly independent, which confers some robustness to our theory. For instance, even if our explanation for the square root price impact $\Delta p \sim V^{1 / 2}$ is erroneous, the rest of the paper still holds. The relations indicated in the Figure 6 are those articulated in the paper, except for the link between Gibrat's law and Zipf's law, which comes from

\footnotetext{
${ }^{14}$ We cannot conclude that the standard deviation on our mean estimate is $0.08(1999-1961+1)^{-.5}$. The estimates are not independent across years, because of the persistence in mutual fund sizes.

${ }^{15}$ Our traders, though intelligent (they try to avoid too high trading costs), are not necessarily hyperrational i.e., they may trade too much, as in Daniel et al. (1999) and Odean (2000).

${ }^{16}$ See Gabaix (1999), Gabaix, Ramalho and Reuter (2003), and Gabaix and Ioannides (2004), and the references therein.
} 


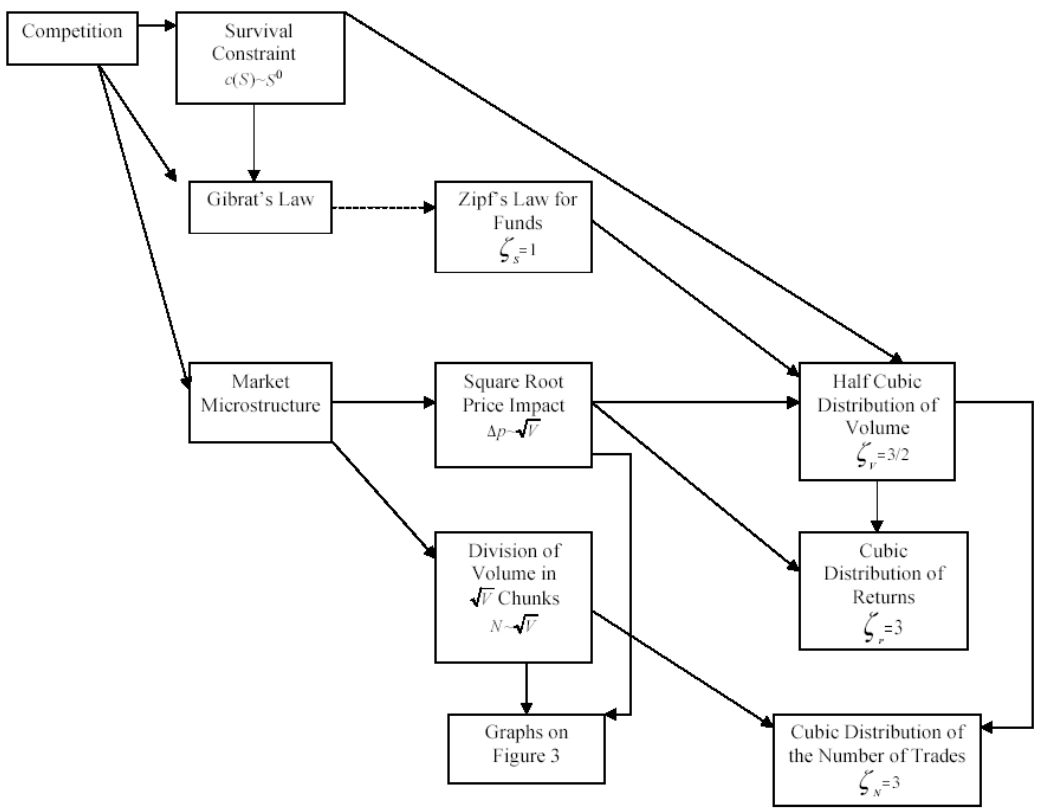

Figure 6: The modular structure of arguments in this paper

Gabaix (1999), Gabaix and Ioannides (2004) and the references therein. It is logically independent of the paper, which is why the link is a dashed line.

\subsection{Notations and some elementary links between exponents}

\subsubsection{Notations}

We call $\zeta_{X}$ the power law exponent of a random variable $X$, i.e. the number such that for a large real $x, P(X>x) \sim x^{-\zeta_{X}}$. If all moments of $X$ are finite, the convention is $\zeta_{X}=+\infty$.

Suppose a large investor wishes to trade a large volume $V$. The large investor divides $V$ into $n$ parts of sizes $q_{1}, \ldots, q_{n}$, so that $V=\sum_{i=1}^{n} q_{i}$. We call $\Delta p$ the price impact due to the entire trade. Over a given time interval, we call $r$ the return, $Q$ the aggregate volume, and $N$ the number of trades. The variables $Q, q_{i}, N, r$ are directly measurable while $V, n$, and $\Delta p$ are not. We have:

$$
\begin{aligned}
r & =\sum_{j \text { traded in that interval }} \Delta p_{j}+u \\
Q & =\sum_{j \text { traded in that interval }} V_{j} \\
N & =\sum_{j \text { traded in that interval }} n_{j}
\end{aligned}
$$

where $u$ reflects other sources of price movement, such as news. 


\subsubsection{Link between aggregate and trader-based exponents}

Our generates power law behaviors for the distribution of 'unobservable' variables such as $V, n$ and $\Delta p$ in (8)-(10). As we show in the proposition below, we relate the power-law exponents for measurable quantities to the unobservable variables, e.g., we show that the measurable power law exponent $\zeta_{Q}=\zeta_{V}$.

Proposition 1 We make the maintained assumption that the $V_{j}$ are independent, that the $n_{j}$ are independent, and $\Delta p_{j}$ and $u$ are independent, and that $\zeta_{\Delta p} \leq \zeta_{u}$. Then

$$
\begin{aligned}
\zeta_{r} & =\zeta_{\Delta p} \\
\zeta_{Q} & =\zeta_{V} \\
\zeta_{N} & =\zeta_{n}
\end{aligned}
$$

Proof. We use results (55) from Appendix A.

Assumption $\zeta_{\Delta p} \leq \zeta_{u}$ means that the power law of returns is due to the features of our model, not to news. The Proposition that the sum of power laws with the same exponent $\zeta$ is also power law with the same exponent $\zeta$ is very general (see Appendix A). In particular, our results will hold under much milder assumptions of independence; as shown in Appendix B, they hold even if there is autocorrelation in market liquidity and trade arrival. For the sake of clarity, we present our results under the simplest assumption of independence.

Next, we discuss an equally simple but more substantive link.

\subsubsection{Link between the exponents of return and volume}

A large literature explores the relation between a trade of size $V$ and a price impact $r=f(V)$. The initial finding of Hasbrouck (1991) is an increasing, concave price impact. This is usefully parametrized by a power law for large volumes $V$ :

$$
\Delta p=h V^{\gamma}
$$

The following Proposition links the exponents of volume, return and price impact.

Proposition 2 Suppose that we have a power law distribution of volumes with exponent $\zeta_{Q}$ and a power law price impact (14) with exponent $\gamma$. Then we have a power law distribution of returns with exponent:

$$
\zeta_{r}=\zeta_{Q} / \gamma
$$

Proof. As $\Delta p=h V^{\gamma}$,

$$
\begin{aligned}
P(\Delta p>x) & =P\left(h V^{\gamma}>x\right)=P\left(V>(x / h)^{1 / \gamma}\right) \\
& \sim\left((x / h)^{1 / \gamma}\right)^{-\zeta_{V}} \sim x^{-\zeta_{V} / \gamma} .
\end{aligned}
$$

so $\zeta_{\Delta p}=\zeta_{Q} / \gamma$. We use Proposition 1 to conclude.

Thus the empirical evidence $\zeta_{Q}=3 / 2$ and $\zeta_{r}=3$ is consistent with a price impact exponent $\gamma=1 / 2$. We will give empirical and theoretical support for $\gamma=1 / 2$ in Section 4 . In the rest of this Section, however, we assume a general $\gamma$. 


\subsection{A main result}

We start with the following puzzle. If each fund $i$ of size $S_{i}$ traded, at random, a volume $V_{i}$ proportional to $S_{i}, V_{i}=a_{i} S_{i}$, then the distribution of volumes would follow $\zeta_{V}=\zeta_{S}$. Then by (7) and Proposition 1, we would have $\zeta_{Q} \simeq 1$, in contrast to the empirical value $\zeta_{Q} \simeq 3 / 2$.

The larger value of $\zeta_{Q}$ compared to $\zeta_{S}$ means that distribution of volumes is less fat-tailed that the distribution of size of investors. This means that large traders trade less often than small traders, or that, when they trade, they trade in volume less than proportional to their sizes. How can we understand this behavior?

Intuitively, a likely reason is that large traders have large price impacts, and have to moderate their trading to avoid paying too large costs of price impacts. This suggest that an important quantity is

$$
c(S)=\frac{\text { Annual amount lost by the fund in price impact }}{\text { Value } S \text { of the assets under management of the fund }}
$$

For example, if funds of size $S$ pay on average $1 \%$ in price impact a year, $c(S)=1 \%$.

The above arguments motivate the following theorem which constitutes a central result of this paper:

Theorem 3 If the following conditions hold:

(i) The size (assets under managements) of large financial market participants follows a power law with exponent $\zeta_{S}$;

(ii) A large volume $V$ has a price impact:

$$
\Delta p \sim V^{\gamma}
$$

with $\gamma>0$

(iii) Funds trade in volumes

$$
V \sim S^{\delta}
$$

for some $\delta>0$;

(iv) Funds adjust trading frequency and/or volume so as to pay an amount transaction costs, relative to their assets:

$$
c(S)=C
$$

(survival constraint);

Then returns and volumes are power law distributed with the exponents:

$$
\begin{gathered}
\zeta_{r}=1+\frac{1}{\gamma}+\frac{\zeta_{S}-1}{\gamma \delta} \\
\zeta_{Q}=1+\gamma+\frac{\zeta_{S}-1}{\delta}
\end{gathered}
$$

We state a useful Corollary.

Corollary 4 In additions to the assumptions of Theorem 3, assume: (i) Zipf's law for investors, $\zeta_{S}=1$, and (ii) the square root price impact, $\Delta p \sim V^{1 / 2}$ (i.e. $\gamma=1 / 2$ ), then returns and volumes are power law distributed with with exponents resp. 3 and 3/2:

$$
\begin{aligned}
\zeta_{r} & =3 \\
\zeta_{Q} & =3 / 2 .
\end{aligned}
$$


We view Theorem 3 and its Corollary 4 as describing the essential features of large movements in market activity. The power law distribution of returns and volumes comes from the power law of large investors (Condition i) and the power law impact of trades (Condition ii). Traders want to avoid paying too much in price impact (Condition iv), which moderates those power laws.

Corollary 4 is relevant, as empirically it seems that $\zeta_{S} \simeq 1$ and $\gamma \simeq 1 / 2$. Section 4 will discuss reasons for $\gamma=1 / 2$. In general, the fatter the tails of the distribution of large agents, the fatter the tails of the distributions of volumes and returns. This may be relevant in highly regulated or immature markets, which might not have $\zeta_{S} \simeq 1$.

We comment on the hypotheses before proceeding to the proof. We have discussed Condition (i) in Section 2.6. The distribution of many other social entities, such as firms, cities and invidual wealth, follows power law distributions.

Condition (ii) is consistent with most empirical work (Hasbrouck 1991, Hasbrouck and Seppi 2002, Plerou et al. 2002, Lillo et al. 2003). We endogenize it in Section 4.

Condition (iii) is a weak technical assumption which we endogenize in Section 3.4. Condition (iii) can be further weakened considerably. The result would still hold, up to logarithmic corrections, if we had $V(S) \sim \exp \left(a S^{\alpha}\right)$ for positives $a, \alpha$. Condition (iii) assumes that $V(S)$ "fast enough" in $S$, i.e., more rapidly than logarithmic.

Condition (iv) means that funds in the upper tail of the distribution pay roughly similar annual price impact costs $c(S)$ reaches an asymptote for large sizes. We provide a foundation for this in Section 3.4. We interpret condition (iv) as an evolutionary "survival constraint". Funds that would have a very large $c(S)$ would have small returns and would be eliminated from the market. The average return $r(S)$ of funds of size $S$ is independent of $S$ (Gabaix, Reuter and Ramalho 2002). Since small and large funds have similarly low ability to outperform the market (see e.g. Carhart 1997), $c(S)$ is also independent of $S$.

We now proceed to the proof of the proposition.

Proof. Each time an investor trades, he incurs a price impact proportional to $V \Delta p$. Given (ii), this cost is $h V^{1+\gamma}$. If $F(S)$ is the an annual frequency of trading, the total annual dollar lost in transactions costs is $F(S) h[V(S)]^{1+\gamma}$, i.e. a fraction

$$
c(S)=F(S) h[V(S)]^{1+\gamma} / S
$$

of the value $S$ of this portfolio. Hypothesis (iii) gives that $V(S)$ and/or $F(S)$ will adjust so as to satisfy:

$$
F(S) \sim S \cdot V(S)^{-(1+\gamma)}
$$

By condition (i) there is a number $G(S) \sim S^{-\zeta_{S}}$ of traders of size greater than $x$. So the density of traders of size $S$ is $\rho(S)=-G^{\prime}(S) \sim S^{-1-\zeta_{S}}$. They trade with frequency $F(S)$ given in (21). Volumes $V>x$ correspond to traders of size $S$ such that $S^{\delta}>x$. Putting all this together:

$$
\begin{aligned}
P(V>x) & \sim \int_{S^{\delta}>x} F(S) \rho(S) d S \sim \int_{S>x^{1 / \delta}} S^{1-(1+\gamma) \delta} S^{-1-\zeta_{S}} d S . \\
& \sim\left[-S^{-(1+\gamma) \delta-\zeta_{S}+1}\right]_{x^{1 / \delta}}^{\infty}=x^{-(1+\gamma)-\left(\zeta_{S}-1\right) / \delta} .
\end{aligned}
$$

which gives (19). The value of $\zeta_{r}$ comes from this and Proposition 2.

One can state a result independent of a particular value of $\gamma$.

Corollary 5 (Reciprocity law between the exponents of returns and volume). Under the assumptions 
of Theorem 3, assuming also $\zeta_{S}=1$ (Zipf's law for large investors), one has, independently of $\gamma$ :

$$
\frac{1}{\zeta_{r}}+\frac{1}{\zeta_{Q}}=1
$$

Proof. This comes from direct calculation from (18) and (19):

$$
\frac{1}{\zeta_{r}}+\frac{1}{\zeta_{Q}}=\frac{1}{1+1 / \gamma}+\frac{1}{1+\gamma}=1
$$

The "reciprocity law" (22) is likely to be particularly robust, as it does not depend on a specific value of $\gamma$. It is verified for our empirical values $\zeta_{r}=3, \zeta_{Q}=3 / 2$.

\subsection{An optimizing model that illustrates Theorem 3}

It is useful to illustrate the "bare-bones" model in Theorem 3 by an economic model. To this end, we introduce a motivation to trade and a reason for why large funds moderate their price impact as in condition (iii). For the motivation for trade, we suppose that fund managers perceive i.i.d. mispricings that are independent across different funds. We will consider a world made of $K$ assets indexed by $i$, but the intuition works for $K=1$ asset. Suppose a manager believes that there is a mispricing $M_{i}$, i.e. that the asset will have excess returns of size $M_{i}$. Without loss of generality, we here take $M_{i}>0$, so that the manager want to buy the asset. Suppose that the fund managers trade on perceived mispricings which occur them $F_{i} \leq F_{i}^{\max }$ times a year ${ }^{17}$. Given a mispricing $M_{i}$, and a volume $V_{i}$ invested in it, the dollar profit from a trade of size $V_{i}$ is the mispricing in excess of the transaction cost: $V_{i}\left(M_{i}-\Delta p_{i}\right)$, where the price impact $\Delta p_{i}$ is

$$
\Delta p_{i}=h_{i} V_{i}^{\gamma} .
$$

In the condition (iii) of Theorem 3: Traders do not wish to lose more than $C \%$ of their funds in transaction costs. Intuitively, this arises because if they follow their instincts to trade too much, their returns may be too small. To capture this, we assume that the trader believes with probability $\pi$ the mispricings are real, while with probability $1-\pi$ the mispricing is a false perception. If the trader's estimated mispricings are reliable his expected returns are

$$
r^{+}=\frac{1}{S} \sum_{i} F_{i} V_{i}\left(M_{i}-\Delta p_{i}\right)
$$

\footnotetext{
${ }^{17}$ Before we proceed, we comment on possible interpretations of $M_{i}$. The simplest is that the trader thinks that the market price is wrong. There is a lot of evidence that investors do not have all the same assessment of the fair valuations, which give rise to the perceived mispricing $M_{i}$. Bagwell (1992) offers early evidence. Diversity in forecasts offers direct evidence for this, and the very high trading volume offers strong indirect suggestion of heterogenous valuations. There is evidence for return predictability (Barberis and Thaler 2003, Hirshleifer 2001, Shleifer 2000) so mispricings get progressively corrected, captured in our model by the $\mu T$ term. A second interpretation for the trading motive $M_{i}$ is that a given point in time, the investor may hold his optimum portfolio. The difference between his current portfolio and his optimum portfolio costs him $\mu$ per unit of time, and is proportional to the dollar amount at stake, $V$. Hence taking $T$ units of time to realize his trade costs him $\mu T V$. So, the derivations in the paper go through with that interpretation. We now proceed to examine the tradeoff that the manager faces between taking advantage of perceived opportunites $M$ and the trading costs.
} 
But if his intuitions are wrong, and markets are efficient, his returns are:

$$
r^{-}=-\frac{1}{S} \sum_{i} F_{i} V_{i} \Delta p_{i}
$$

The traders has an objective function $U$ on returns, so that his expected utility is:

$$
W=\pi U_{\Gamma, \underline{r}}\left(r^{+}\right)+(1-\pi) U_{\Gamma, \underline{r}}\left(r^{-}\right) .
$$

We will consider the class of utility functions:

$$
U_{\Gamma, \underline{r}}(r)=u(r+\underline{r})-Z e^{-\Gamma(r+\underline{r})} .
$$

for some positive $Z, \Gamma$ and some increasing weakly concave function $u . U_{\Gamma, \underline{r}}$ is increasing and concave and satisfy:

$$
\begin{aligned}
\lim _{\Gamma \rightarrow \infty} U_{\Gamma, \underline{r}}(r) & =u(r+\underline{r}) \text { if } r+\underline{r}>0 \\
& =-\infty \text { if } r+\underline{r}<0
\end{aligned}
$$

In other terms, the trader has a standard utility function for returns $r$ above a threshold $-\underline{r}$, but wants to avoid getting returns below $-\underline{r}$. We can think of $-\underline{r}<0$ as some low underperformance threshold below which the fund managers lose their job. $\Gamma$ is an aversion to underperformance.

We state the Proposition.

Proposition 6 In the setup above, suppose that the "cost cap" $\underline{r}$ is low enough, so that the survival constraint (iv) in Theorem 3 holds (c.f. Eq. (33)). Then, as the aversion to underperformance $\Gamma$ become very large, hypothesis (ii) and (iii) of the Theorem 3 hold for each stock $i$, with

$$
\delta=1 /(1+\gamma) .
$$

The size of trades is:

$$
V_{i}=k\left(\frac{M_{i}}{h_{i}}\right)^{1 / \gamma} S^{1 /(1+\gamma)}
$$

for a constant $k$ given in (34). The total amount spent in transaction costs is the value of the portfolio times $\underline{r}$.

Thus this economic model generates the conditions (iii) and (iv) of Theorem 3. It makes a prediction (25) on the value of $\delta$ that could in principle be tested. The volume traded increases with the size of the trader, but a in less than linearly. The elasticity $\partial \ln V / \partial \ln S=1 /(1+\gamma)$ is higher in markets in markets with low elasticity of price impact $\gamma$.

We also see that, in term of Theorem 3, if the constraint (iv) holds for the whole portfolio, it will hold stock by stock too.

It was expected that, as in (26), funds trade in larger volume when they are bigger, when the perceived profit opportunities $M_{i}$ are larger, and the prefactor $h_{i}$ of price impact is small.

Proof. We study directly the case $\Gamma=\infty$, as the problem is continuous in $\Gamma$. The trader's problem is:

$$
\max _{F_{i}, V_{i}} \sum_{i=1}^{K} F_{i} V_{i}\left(M_{i}-\Delta p_{i}\right)
$$




$$
\begin{aligned}
\text { s.t. } \sum_{i=1}^{K} F_{i} V_{i} \Delta p_{i} & \leq \underline{r} S, \\
F_{i} & \leq F_{i}^{\max }, \text { and } \\
\Delta p_{i} & =h_{i} V_{i}^{\gamma}
\end{aligned}
$$

The Lagrangian is:

$$
\mathcal{L}=\sum_{i} F_{i} V_{i}\left(M_{i}-\Delta p_{i}\right)+\lambda\left(\underline{r} S-\sum_{i} F_{i} V_{i} \Delta p_{i}\right)+\sum_{i} \kappa_{i}\left(F_{i}^{\max }-F_{i}\right)
$$

and the first order conditions are:

$$
\begin{aligned}
\frac{1}{V_{i}} \frac{\partial \mathcal{L}}{\partial F_{i}} & =M_{i}-(1+\lambda) h_{i} V_{i}^{\gamma}-\frac{\kappa_{i}}{V_{i}}=0 \\
\frac{1}{F} \frac{\partial \mathcal{L}}{\partial V_{i}} & =M_{i}-(1+\lambda)(1+\gamma) h_{i} V_{i}^{\gamma}=0
\end{aligned}
$$

Eq. (31) gives $V_{i}>0$, and (30)-(31) give $\kappa_{i}=(1+\lambda) \gamma h_{i} V_{i}^{\gamma}>0$, i.e. (29) binds. So:

$$
V_{i}^{\gamma}=\frac{M_{i}}{(1+\lambda)(1+\gamma) h_{i}}
$$

The left-hand side of (28) is:

$$
H(\lambda)=[(1+\gamma)(1+\lambda)]^{-1-1 / \gamma} \sum_{i} F_{i}^{\max } M_{i}^{1+1 / \gamma} h_{i}^{-1 / \gamma}
$$

and (28) binds iff $H(0)>\underline{r} S$, i.e.

$$
\underline{r}<\frac{1}{S}(1+\gamma)^{-1-1 / \gamma} \sum_{i} F_{i}^{\max } M_{i}^{1+1 / \gamma} h_{i}^{-1 / \gamma}
$$

As we assume this, $\lambda$ solves $H(\lambda)=\underline{r} S$. With (32), this yields (26) where:

$$
k=\left[\frac{\underline{r}}{\sum_{i} F_{i}^{\max } M_{i}^{1+1 / \gamma} h_{i}^{-1 / \gamma}}\right]^{1 /(1+\gamma)}
$$

The total cost constraint for stock $i$ is $C_{i}=F_{i}^{\max } h_{i} V_{i}^{1+\gamma} / S$, i.e.

$$
C_{i}=\frac{F_{i}^{\max } M_{i}^{1+1 / \gamma} h_{i}^{-1 / \gamma}}{\sum_{j} F_{j}^{\max } M_{j}^{1+1 / \gamma} h_{j}^{-1 / \gamma}} \underline{r}
$$

In particular $\sum_{i} C_{i}=\underline{r}$. 


\section{The square root of price impact of trades: Evidence and a possible explanation}

At this stage, we understand how a power law price impact of trading, and a power law distribution of traders sizes, gives rise to power law distributions of volume and returns. We now explore why a particular value of the price impact, $\gamma=1 / 2$, is favored on empirical and theoretical grounds. The initial motivation is Proposition 2, which shows that a square root impact $\gamma=1 / 2$ precisely gave the right link between $\zeta_{r}=3$ and $\zeta_{Q}=3 / 2$. So we first present empirical evidence that supports $\gamma=1 / 2$, and then propose a model that can explain this particular value.

\subsection{Evidence on the square root law of price impact}

We provide evidence here for the $\gamma=1 / 2$ in (14), i.e. a price impact function:

$$
\Delta p \sim V^{1 / 2}
$$

We start from the benchmark where, in a given time interval, $J$ i.i.d. blocks are traded, with volumes $V_{1}, \ldots, V_{J}$, with i.i.d. signs $\varepsilon_{j}= \pm 1$. The aggregate volume is $Q=\sum_{j=1}^{J} V_{j}$, and the aggregate return is:

$$
r=u+h \sum_{j=1}^{J} \varepsilon_{j} V_{j}^{1 / 2}
$$

where $u$ is some other source of price movement, assumed to be uncorrelated. Then,

$$
\begin{aligned}
E\left[r^{2} \mid Q\right] & =\sigma_{u}^{2}+h^{2} E\left[\sum_{j} V_{j}+\sum_{j \neq k} \varepsilon_{j} \varepsilon_{k} V_{j} V_{k} \mid Q\right] \\
& =\sigma_{u}^{2}+h^{2} Q+0 .
\end{aligned}
$$

We gather:

$$
E\left[r^{2} \mid Q\right]=\sigma_{u}^{2}+h^{2} Q
$$

Figure 7 shows the empirical result ${ }^{18}$. It reveals an affine relation, rather than any clear sign of concavity or convexity. A formal test that we detail in Appendix F confirms this. We thus name (35) as a good candidate for an empirical law, and now propose a theory for it.

\subsection{Sketch of our explanation for the square root price impact}

We now present a sketch of our explanation for (35) before developing it further. The basic microstructure models (e.g. Kyle 1985) give a linear ${ }^{19}$ price impact $\Delta p \sim V$. Virtually all the empirical evidence (starting with Hasbrouck 1991), however, shows a concave price impact. Put succinctly, the argument is the following. In our model, a trader who is willing to wait for an amount of time

\footnotetext{
${ }^{18}$ The construction of the confidence interval requires special care, as $r^{2}$ has infinite variance.

${ }^{19}$ A concave price-volume is given by Seppi (1990), Barclay and Warner (1993), and Keim and Madhavan (1996). These models do not predict a square root, however.
} 


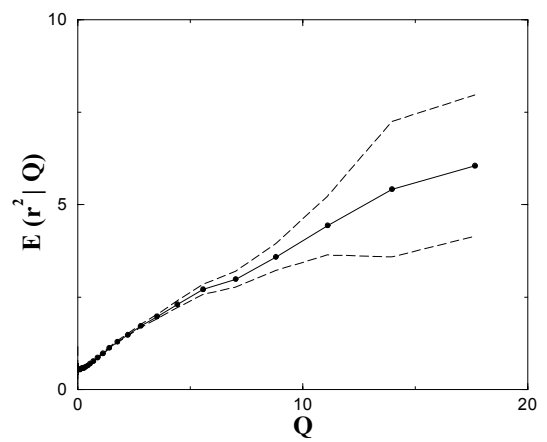

Figure 7: Conditional expectation $E\left[r^{2} \mid Q\right]$ of the squared return $r^{2}$ given the volume $Q$. The bands represent $95 \%$ confidence intervals. The theory predicts, for large $Q$ 's, a relation $E\left[r^{2} \mid Q\right]=\alpha+\beta Q$, the "square root" price impact of volume. $r$ is in units of standard deviation, and $Q$ in units of the first moment. The time interval is $\Delta t=15 \mathrm{~min}$, and the results are averaged over $N=116$ liquid stocks. Formal tests reported in Appendix $\mathrm{F}$ show that one cannot reject $E\left[r^{2} \mid Q\right]=\alpha+\beta Q$ large enough $(Q \geq 3)$.

$T$ to realize his buy trade will incur a price impact:

$$
\Delta p=\frac{V}{T}
$$

so that Kyle (1985)'s linearity of the price impact in $V$ holds, but for a given $T$. But more patient traders who are willing to wait for a larger time $T$, will be have smaller price impact. Suppose that asset's price is increasing at a rate $\mu$. The total benefit from the trade of size $V$ will be:

$$
B=V(M-\mu T-\Delta p)
$$

The optimal $\Delta p$ maximizes $B$ subject to (37), so that

$$
\Delta p=\arg \max _{\Delta p} V\left(M-\mu \frac{V}{\Delta p}-\Delta p\right)
$$

which gives

$$
\Delta p=(\mu V)^{1 / 2} \sim V^{1 / 2}
$$

and $T \sim V^{1 / 2}$. In words, the price impact of a trade of size $V$ scales like less than linear $(\Delta p \sim V)$ because large traders are willing to wait for a longer time $T$ to moderate their price impact. In other words, in order to execute a trade of size $V$, traders are on the average more patient and wait for a time $T \sim V^{1 / 2}$, so that Eq. (37) gives Eq. (35). We now proceed to the model that gives (37). 


\subsection{A microstructure model for the power law impact of a block}

We will present a search model for the way in which a trader finds counterparts for a large trade. Our model is a search model. Other models include He and Wang (1995), Saar (2001), Vayanos (2001), Duffie, Garleanu and Pedersen (2002).

\subsubsection{The behavior of liquidity providers}

We will present a model where a seller of size $s_{i}$ who received from the buyer a price concession $\Delta p$ is willing to supply a number of shares

$$
q_{i}=\alpha s_{i}|\Delta p|
$$

where $\alpha$ is some proportionality factor. This linear supply could be the linearization of a host of models. We present one such model in Appendix D.

\subsubsection{Permanent vs Transitory price impact}

In most microstructure analyses, the price impact has a temporary and a permanent component. The typical implementation is:

Assumption $\mathbf{A}$ on the temporary and permanent price impact. If the mid-quote price before the trade was $p_{t-1}$, and the transaction price was $p_{t}^{\text {Trade }}=p_{t-1}+\Delta p_{t}$, then the next mid-quote price is on average $p_{t+1}=p_{t-1}+b \Delta p_{t}$ with $b \in(0,1)$.

Various ways to model this are offered in the literature (Biais et al. 2002, O'Hara 1995). Here weadopt a very simple justification for this. Our results would not change if $b_{t}$ had a seasonal (e.g., time of the day) component, or was random with power law $\zeta_{b} \geq 3$.

Proposition 7 Suppose that in the model there are risk neutral arbitrageurs who think that, when a traded price differs from the previous quoted price, the trade comes from probability $b$ from an informed, risk neutral trader, and with probability $1-b$ from uninformed trader. Then, they will will enforce Assumption A above.

Proof. Call $f_{t}$ the fundamental price, $\mathcal{F}_{t}$ the information at time $t$, and $I_{t}=1$ if the trade is informed and 0 otherwise, so that $b=P\left(I_{t}=1\right)$. Arbitrageurs impose $p_{t-1}=E\left[f_{t-1} \mid \mathcal{F}_{t-1}\right]$, and the after-trade price is:

$$
\begin{aligned}
p_{t+1} & =E_{t}\left[f_{t+1} \mid p_{t}^{\text {Trade }} \text { and } \mathcal{F}_{t-1}\right] \\
& =b E_{t}\left[f_{t+1} \mid p_{t}^{\text {Trade }}, I_{t}=1 \text { and } \mathcal{F}_{t-1}\right]+(1-b) E_{t}\left[f_{t+1} \mid p_{t}^{\text {Trade }}, I_{t}=1 \text { and } \mathcal{F}_{t-1}\right] \\
& =b\left(p_{t-1}+\Delta p\right)+(1-b) p_{t-1} \\
& =p_{t-1}+b \Delta p .
\end{aligned}
$$

The above justification is not particularly interesting, as it does not make any prediction. Its only merit is to close our model in a simple way.

\subsubsection{The behavior of the large trader}

We consider a large trader $A$ who wants to buy a large number of shares $V$. To accomplish the trade, the trader will search for counterparts. When all the counterparts are found, the trade is 
executed, i.e. the trades with the counterparts are executed at the same time. Our description is a stylization of the "upstairs" market, and is in the spirit of other search models in the literature.

At time $t=0$ trader $A$ decides to buy a quantity $V$ and looks for a counterpart. Liquidity providers, indexed by $i$, are found stochastically ${ }^{20}$. Because of (38), liquidity providers are willing to sell the active trader a quantity of shares $q_{i}=\alpha s_{i} \Delta p$. So after a time $t$, the active trader $A$ can buy

$$
\sum_{\text {traders } i \text { that have appeared between } 0 \text { and } t} \alpha s_{i} \Delta p .
$$

The search process stops when the desired quantity is reached, i.e. at a time $\widetilde{T}$ such that ${ }^{21}$

$$
\sum_{i \text { arrives between } 0 \text { and } \widetilde{T}} \alpha s_{i} \Delta p=V
$$

Let $f$ denote the $\left(\right.$ Poisson $^{22}$ ) frequency of arrival of liquidity providers, $\bar{s}$ their average size, and the average time for realizing the trade be $T=E[\widetilde{T} \mid \Delta p, V]$. Taking expectations on (39), we obtain $f T \alpha \bar{s} \Delta p=V$, and:

$$
T=\frac{V}{\alpha f \bar{s} \Delta p}
$$

The trader's goal is to minimize two quantities: the execution time $T$, and the execution cost $|\Delta p|$. From above, his objective function for the given trade is:

$$
B=V(M-\mu T-\Delta p)
$$

Indeed, as above, the trader expects the asset to have excess returns $M$. But after a wait of $T$, the price has gone up by $\mu T$, so that the remaining mispricing is only $M-\mu T$. The total dollar profit $B$ is the realized excess return $M-\mu T$ minus the price concession $\Delta p$, times the dollar volume transacted $V$. Given (41) and (40)

$$
\Delta p=\arg \max _{\Delta p} V\left(M-\mu \frac{V}{\alpha f \bar{s} \Delta p}-\Delta p\right) .
$$

This give $\Delta p \sim V^{1 / 2}$, the square root price impact (35). We present this formally:

Proposition 8 In the above setting, $\gamma=1 / 2$. Traders incur the square root law of price impact:

$$
\Delta p \sim V^{1 / 2},
$$

wait for an mean amount of time

$$
T \sim V^{1 / 2}
$$

\footnotetext{
${ }^{20} \mathrm{An}$ interpretation is that the active trader $A$ has, perhaps via his broker, calls successively potential buyers. After time $t$, the number of potential liquidity providers are proportional to $t$.

${ }^{21}$ Here we omit integer constraints. Taking them into account would complicate but not change the analysis.

${ }^{22}$ To increase descriptive realism, we could assume that the Poisson arrival rate $f$ itself increases with the price offered $|\Delta p|$, as in $f(\Delta p)$. It is easy to verify that our results for the power laws will not change if the function $f$ increases, for large $|\Delta p|$, like less than a power law (e.g. the arrival rate tends to saturation maximal value as $|\Delta p| \rightarrow \infty$, or say $f(\Delta p) \leq a \ln (b+|\Delta p|)^{k}$ for some finite $\left.a, b, k\right)$.
} 
and trade with a mean number of liquidity providers

$$
n \sim V^{1 / 2}
$$

The full expressions are:

$$
\begin{aligned}
\Delta p & =\left(\frac{\mu V}{\alpha f \bar{s}}\right)^{1 / 2} \\
E[\widetilde{T} \mid V] & =\left(\frac{V}{\alpha f \mu \bar{s}}\right)^{1 / 2} \\
E[n \mid V] & =\left(\frac{f V}{\alpha \mu \bar{s}}\right)^{1 / 2} .
\end{aligned}
$$

Proof. The loss function (39) gives (44) while (45) comes from (40). As liquidity providers

arrive with frequency $f$, we have $E[n \mid V]=f E[\widetilde{T} \mid V]$.

So large traders create large price impacts, and have to accept a delay in the execution of their trades. This is qualitatively consistent with the stylized facts found in the empirical literature : the price impact $|\Delta p|$ is an increase and concave function of the trade size $V$ (Hasbrouck 1991, Holthausen et al. 2000, who find that a large part of the impact is permanent), impatient traders (high cost of time $\mu$ ) will have a bigger price impact $\Delta p$ (Chan and Lakonishok 1993, 1995, Keim and Madhavan 1996, 1997, Lo, MacKinlay and Zhou 1997, Breen, Hodrick, Korajczyk 2002). Our theory gives a precise quantitative hypothesis for this price impact and delay, $\Delta p \sim V^{1 / 2}$ and $T \sim V^{1 / 2}$. Future research might examine directly whether those square root predictions (for large trades) hold in the data.

\subsection{The distribution of individual trades $q$ vs distribution of target vol- umes $Q$}

Theorem 3 gives reasons for distribution of target volumes with $\zeta_{V}=3 / 2$. The "upstairs" mechanism above provides a way to divide them into a several smaller trades of size $q_{i}$. We describe the resulting distribution below ${ }^{23}$.

Proposition 9 In the search process above, if distribution of target volumes has power law exponent $\zeta_{Q}>1$, and the distribution of the sizes of liquidity providers has power law exponent $\zeta_{s}=1$, then the distribution of individual trades has power law exponent

$$
\zeta_{q}=\zeta_{Q}
$$

Proof. We will actually derive the Proposition in a more general form, as here we assume the following supply function for the number of shares supplies by a liquidity provider of size $s_{i}$ to a large trader who wants to buy a volume $V$ :

$$
q_{i}=\alpha s_{i} \Delta p=\alpha^{\prime} s_{i} V^{\gamma}
$$

for a $\gamma \in(0,1)$, not necessarily $\gamma=1 / 2$. Individual trades of size $x$ come from large trades of size $V(>x)$. There is a density $\rho(V) \sim V^{-\left(\zeta_{V}+1\right)}$ of them. Each large volume $V$ generates a number

\footnotetext{
${ }^{23}$ More technical, rigorous results are given in Gabaix, Gopikrishnan, Plerou and Stanley (2003b).
} 
$n(V)=V / E[q] \sim V^{1-\gamma}$ of trades. So we find:

$$
\begin{aligned}
P\left(q_{i}>x\right) & \sim \int_{V>x} \rho(V) n(V) P\left(s_{i} V^{\gamma}>x\right) d V \\
& \sim \int_{V>x} V^{-\left(\zeta_{V}+1\right)} \cdot V^{1-\gamma} P\left(s_{i} V^{\gamma}>x\right) d V .
\end{aligned}
$$

As the liquidity providers' size distribution is power law with exponent $\zeta_{s}=1$,

$$
P\left(s_{i} V^{\gamma}>x\right)=P\left(s_{i}>x / V^{\gamma}\right) \sim\left(x / V^{\gamma}\right)^{-1}
$$

and

$$
\begin{aligned}
P\left(q_{i}>x\right) & \sim \int_{V>x} V^{-\left(\zeta_{V}+1\right)} V^{1-\gamma}\left(\frac{x}{V^{\gamma}}\right)^{-1} d V \\
& =x^{-1} \int_{V>x} V^{-\zeta_{V}} d V \sim x^{-1} \cdot x^{-\left(\zeta_{V}-1\right)}=x^{-\zeta_{V}}
\end{aligned}
$$

so we have $\zeta_{q}=\zeta_{V}$.

A very similar proof would show that $\zeta_{q}=\zeta_{V}+\left(\zeta_{s}-1\right)(1-\gamma)$ for $\zeta_{s} \neq 1$.

\subsection{An optimizing intertemporal model that joins power laws and mi- crostructure}

We now join our model of intertemporal trading with the model of Section 3.4, and our microstructure model of Section 4.3. It turns out that a key ingredient is price leakages. In their absence, traders would trade very slowly, creating very little price impact. Leakage is an important concern in trading large blocks. Keim and Madhavan (1996) shows that in their sample leakage more than doubles the price impact of large trades ${ }^{24}$.

We assume that, during the search process for the block trade by the large trader $A$, the price increases at a rate $\mu+\nu$, where $\nu$ represents the rate of leakage. There is leakage when an investor $B$, after hearing that $A$ is going to buy, try to "front run" $A$ and buy before him. Given that leakage affects the execution price, it should be included in the transaction costs, i.e. count in the constraint (iii) of Theorem 3.

For the ideas above, we propose the following simplified formalization. Suppose that with a probability $\pi$ liquidity provider $i$ will front run $A$, i.e. trade before him. Liquidity provider $i$ will have a mean price impact $\overline{I\left(s_{i}\right)}$. Hence the price pressure associated with trading the block is an increase of the price per unit of time:

$$
\nu=\pi \overline{I\left(s_{i}\right)} f,
$$

where $f$ is the number of liquidity providers contacted per unit of time. The fact that $\nu>0$ is important, but the exact expression of $\nu$ does not very important.

We adopt the same structure of beliefs and objective function as in Section 3.4. In the trader's opinion, the dollar profit from a trade of size $V$ is: $V(M-(\mu+\nu) \Delta p)$. So if he has true intuitions about the market, his the excess returns are:

$$
r^{+}=F \cdot V(M-(\mu+\nu) T-\Delta p) / S
$$

If his intuitions about the market are false, his excess returns are:

\footnotetext{
${ }^{24}$ Without leakage the price impact if $4.3 \%$, while with leakage it is $10.2 \%$.
} 


$$
r^{-}=-F \cdot V(\nu T+\Delta p) / S
$$

Hence, the price impact includes not only $\Delta t$, but also the price increment due to leakage $\nu T$.

The trader maximizes $W$ in (24) subject to $F \leq F^{\max }$ and (39), which we rewrite as:

$$
\Delta p=a \frac{V}{T}
$$

The solution is given by the next Proposition.

Proposition 10 In the market setup above, we assume that the trader maximizes the objective function $W$ given in (24). In the regime where $\Gamma \gg 1$ (large aversion to underperformance) and $M \gg S^{1 / 3} \mu$ (which can be more concretely expressed $M \gg \mu T$ for the optimal solution), the solution of problem above yields $V \sim S^{\delta}$ with $\delta=2 / 3$. Thus, the conditions of Theorem 3 hold with $\delta=2 / 3$ and the square root price impact $\gamma=1 / 2$.

Proof. It is easy to see that the constraints bind. Consider the limit $\mu=0$. The problem becomes:

$$
\begin{gathered}
\max _{\Delta p, V} F^{\max } V M-\underline{r} S \\
\text { s.t. } F^{\max }\left(\nu a \frac{V}{\Delta p}+\Delta p\right) V=\underline{r} S
\end{gathered}
$$

Optimizing over $\Delta p$, we get $\Delta p=(\nu a V)^{1 / 2}$, and (48) gives $2 V^{3 / 2} F^{\max }(\nu a)^{1 / 2}=\underline{r} S$, hence $V \sim$ $S^{2 / 3}$.

The proof is now complete. In addition, it may be instructive to see which variables matter for the limit $\mu \rightarrow 0$. For this define $x=(\nu a V)^{1 / 2} / \Delta p, B=\underline{r} F^{\max -1}(\nu a)^{-1 / 2}, \eta=B^{1 / 3} M^{-1} S^{1 / 3}(a / \nu)^{1 / 2} \mu$, and $y=B S V^{-3 / 2}$. The problem becomes:

$$
\max y^{-2 / 3}-\eta x y^{-1} \text { s.t. } x+x^{-1}=y
$$

This forces $y \geq 2$, and leads to $x=\xi(y)=\left(y-\left(y^{2}-4\right)^{1 / 2}\right) / 2$, and $y(\eta)=\arg \max y^{-2 / 3}-$ $\eta \xi(y) y^{-1}$.We have $y(0)=2$, and the solution is continuous in $\eta$. Thus we get $y(\eta)=2+o(\eta)$, i.e. $V=S^{2 / 3}\left(1+o\left(\mu \underline{r}^{1 / 3} S^{1 / 3} M^{-1}\right)\right)$.

Hence our predictions are confirmed by this framework. The cubic law arises if large traders are forced to trade quickly because of price leakage.

\subsection{Some comments on the model}

\subsubsection{Arbitrage}

Our model is does not leave scope for short-term arbitrage, as the trading decisions are unpredictable. However, it offers some scope for predictability of long term returns. Indeed as volume moves prices without necessarily informational reasons, prices deviate from fundamentals, and they will revert in the long term. There is a evidence for this phenomenon, see e.g. Campbell and Shiller (1988). So our model requires limited arbitrage of long term returns. There are good reasons why long term arbitrage is difficult, see e.g. Shleifer and Vishny (1997) and Shleifer (2000). 


\subsubsection{Plausibility of very large volumes and price impact}

The literature finds a big impact of big traders. Chan and Lakonishok $(1993,1995)$ find a range of 30-100 bps, Keim and Madhavan (1996), looking at smaller stocks, find 400 bps. There are also many colorful examples of large investors moving the market. Some are reported by Corsetti, Pesenti and Roubini (2002), Coyne and Witter (2002) and the Report on the 1987 crash (United States' Presidential Task Force on Market Mechanisms 1988).

As it is difficult to detect exogenous trades, measuring a price impact is difficult. There is a more "intuitive" way to convince oneself that a large fund is likely to move prices. The typical yearly turnover of a stock is 50\% (Lo and Wang 2001), so that with 250 trading days per year, its daily turnover is $0.5 / 250=0.2 \%$ of the shares outstanding. Consider a moderate size fund, e.g. the 30th largest fund. At the end of 2000 , such a fund held $0.1 \%$ of market ${ }^{25}$. So, on the average, it will hold $0.1 \%$ of the capitalization of a given stock. So if the fund wants to sell its holdings, it will create an additional $0.1 \%$ in turnover of stock, while the regular turnover is $0.2 \%$ per day, i.e., the fund will have to absorb half of the daily turnover. So, the size of the desired trade of this fund is quite important compared to the normal turnover. This supports the idea that large funds are indeed large for liquidity of the market, and supports the assumption that big traders will pay attention to their trading strategy to moderate their price impact.

\section{$5 \quad$ Assessing some further empirical predictions of the model}

\subsection{Contemporaneous behavior of several measures of trading activity}

\subsubsection{Definitions}

The above model proposes an explanation for the power laws of financial market activity. We now show how it correctly explains another series of patterns in trading activity, pertaining to the joint distributions of returns, volumes, and their variants. More precisely, we fix a time interval $\Delta t=15 \mathrm{~min}$. We will consider the return $r_{t}$, the volume ${ }^{26} Q_{t}$ and number of trades $N_{t}$ in interval $(t-\Delta t, t]$. We also partition those quantities in buys and sells. Suppose each trade $i$ occurring at time $i$, with a quantity of shares exchanged $q_{i}$, and define $\varepsilon_{i}=+1$ is the trade can be identified as buy initiated, $\varepsilon_{i}=-1$ if it was sell-initiated, $\varepsilon_{i}=0$ if no identification is possible ${ }^{27}$. We define $N_{t}^{\prime}$ as the net number of trades, i.e. number of "buy" trades minus the number of "sell" trades. Finally we define $Q_{t}^{\prime}$ as the net volume, i.e. number of shares exchanged that come from a buy order minus

\footnotetext{
${ }^{25}$ It had $\$ 19$ billion in assets under management. The total market capitalization of The New York Stock Exchange, the NASDAQ and the American Stock Exchange was $\$ 18$ trillion.

${ }^{26}$ The dollar value of the shares traded, or the number of the shares traded divided by the number of shares outstanding give the same results, as they are proportional in the short term.

${ }^{27}$ We us the Lee-Ready (1991) algorithm. We identify buyer and seller initiated trades using the bid and ask quotes $P_{\mathrm{B}}(t)$ and $P_{\mathrm{A}}(t)$ at which a market maker is willing to buy or sell respectively. Using the mid-value $P_{\mathrm{M}}(t)=$ $\left(P_{\mathrm{A}}(t)+P_{\mathrm{B}}(t)\right) / 2$ of the prevailing quote, we label a transaction buyer initiated if $P(t)>P_{\mathrm{M}}(t)$, and seller initiated if $P(t)<P_{\mathrm{M}}(t)$. For transactions occurring exactly at $P_{\mathrm{M}}(t)$, we use the sign of the change in price from the previous trade to determine whether the trade is buyer or seller initiated, while if the previous transaction is at the current trade price, the trade is labelled indeterminate. We use the prevailing quote at least $5 \mathrm{~s}$ prior to the trade. $17 \%$ of the trades are left indeterminate.
} 
number of shares exchanged that come from a sell order. Formally:

$$
\begin{aligned}
N_{t} & =\sum_{\text {trade } i \text { happening in }(t-\Delta t, t]} 1 \\
Q_{t} & =\sum_{\text {trade } i \text { happening in }(t-\Delta t, t]} q_{i} \\
N_{t}^{\prime} & =\sum_{\text {trade } i \text { happening in }(t-\Delta t, t]} \varepsilon_{i} \\
Q_{t}^{\prime} & =\sum_{\text {trade } i \text { happening in }(t-\Delta t, t]} \varepsilon_{i} q_{i}
\end{aligned}
$$

We compute the graphs $E[Y \mid X]$ for many combinations of $X, Y=r, Q, Q^{\prime}, N, N^{\prime}, N^{\prime} / N$, $Q / N$. The choice was guided by an assessment of how informative such a graph would be, and how non-trivial the shape predicted by the theory would be. The model reproduces all the shapes of those graphs, as can be seen in Figure 8 below.

We use simulations to plot the model's predictions. We compare the empirical and theoretical conditional expectations $E[Y \mid X]$, for $X, Y=Q, N, Q^{\prime}, N^{\prime}, r, N^{\prime} / N, r^{2}, Q / N$. We show our results only for selected pairs of $X, Y$ that seem nontrivial.

\subsubsection{Comparison between theory and facts}

In Figure 8a-e we present the empirical values on the left panel, and the model's predictions on the right panel. We obtained the model's predictions using Monte Carlo simulations detailed in Appendix E. The graphs show that the model broadly matches ${ }^{28}$ all the $E[Y \mid X]$ that we construct empirically. Consider for example $E\left[r \mid Q^{\prime}\right]$ in Figure 8a. Our model reproduces on the left panel the increasing, concave shape (in the positive region). The aggregation over several trades flattens the shape of the price impact function. Plotting the symmetrical version, $E\left[Q^{\prime} \mid r\right]$, yields a surprising finding. The empirical graph, in Figure $8 \mathrm{~b}$ is now linear. The model matches this in the right panel of Figure 8b. The cause of the linearity, again, is the aggregation over several trades.

The model predicts that periods of large volume are periods when large traders execute trades in smaller pieces, so that, for a large buy trade, one observes large number of buy smaller trades. A compact way to say that is that $N$ is high, and $\left|N^{\prime} / N\right|$ is high too when the volume is high $^{29}$. This is expressed in Figure 8c.

It is an important feature of the model that large trades beget more trades. Indeed, in our model

$$
\left|N^{\prime}\right| \sim N
$$

for large $N$ and is dominated by one large fund manager who desires to trade a volume $V_{j}$, and creates a number of orders $V_{j}^{1 / 2}$, so that $n_{j}, N$, and $\left|N^{\prime}\right|$ have the same order of magnitude, $V_{j}^{1 / 2}$. Relation (53) means that most trades have the same sign, i.e., move the price in the same direction,

\footnotetext{
${ }^{28}$ Given we have 1.5 million observations, it is likely that statistical tests would show that the fit is not perfect. Our intention here is just to illustrate that the fit is good. To obtain a perfect fit, one would need to complicate the model, in particular to introduce autocorrelation in the propensity to trade. Here we chose to show that the simple, non-optimized, i.i.d. version of our model can get a pretty good fit.

${ }^{29} \mathrm{~A}$ very different relationship would happen under another plausible model. Suppose that there are $N$ trades with i.i.d. signs $\left(\varepsilon_{i}\right)_{i=1 \ldots N}$ and sizes $\left(V_{i}\right)_{i=1 \ldots N}$, so that $Q^{\prime}=\sum_{i=1}^{N} \varepsilon_{i} V_{i}$. Then a large $N$ corresponds to an $N^{\prime}=$ $\sum_{i=1}^{N} \varepsilon_{i}=O(\sqrt{N})$, so that $N^{\prime} / N=O(1 / \sqrt{N})$ is small. Likewise a large $Q^{\prime}$ corresponds in part to a large $N$, and a small $\left|N^{\prime} / N\right|$, counterfactually.
} 
the sign of the trade of the large fund manager. Equation (53) is indeed consistent with empirical data shown in Figure 8d. This contrasts with a simple alternative model where each desire to trade would create only one trade, as in a competitive market. In this alternative model we would have $N^{\prime}=\sum_{i=1}^{N} \varepsilon_{i}$, where $\varepsilon_{i}= \pm 1$, leading to $\left|N^{\prime}\right| \sim N^{1 / 2}$ in the tail events, or $E\left[N \mid N^{\prime}\right] \sim N^{\prime 2}$ in contrast to the data in Figure 8d. Figure 8e supports the view that in periods of high volume imbalance $V^{\prime}$, most trades change the price in the same direction. Indeed, the data and the model exhibit a similar sharp transition of $N^{\prime} / N$ as $V^{\prime}$ changes sign.

We tested other cases too and we find good agreement between the theory and empirical data ${ }^{30}$. Here, we report the curves with the shapes we deem most informative. Given that the model was not constructed to match those facts, we view this as a very reassuring feature of the model.

\subsection{Some untested predictions}

The model makes a number of untested predictions, which we present in this Section.

- As per equation (21), a large fund of size $S$ will do "price-moving" block trades ${ }^{31}$ of size $V \sim S^{\delta}$ with a frequency that depends on size as $S / V^{1+\gamma}$, where $\gamma$ is the exponent of the price impact (14).

- Expression (26) for the volume traded in an individual stock, in particular the dependence in $S$ and the prefactor of the price impact $h_{i}(23)$

- The expressions (44)-(46) for the price impact, the time to execution, and the number of trades. In particular, it would be interesting to test their "square root" dependence in $S$, and the differential impact of $\mu$ (the impatience of the trader), $\alpha, f, \bar{s}$.

- The linear supply function (38), saying that a trader willing to wait an amount of time $T$ and to pay a price increment $\Delta p$, will get an amount of shares $S(\Delta p, T)$ that will be proportional to $\Delta p \cdot T$ in the limit of large $\Delta p$ and $T$.

- We anticipate the values of the four trading exponents $\alpha, \beta, \kappa$ and $\tau$ in Appendix B to be 0 , 1,0 and 1 respectively.

Hence our theory makes predictions about the value of 24 power law exponents ${ }^{32}$. We can make also graphical predictions $E[Y \mid X]$ graphs that we did not build ${ }^{33}$, which yields 56 predictions. We leave the investigation of those values to future research.

\section{Related literature}

\subsection{Alternative theories}

The general theme is that most theories (certainly those that we are aware of) do not explain (and cannot explain with a simple modification) the cubic and half-cubic laws. At best, they have free parameters which can be artificially tuned to replicated the $\zeta_{r}=3$.

\footnotetext{
${ }^{30}$ The graphs we tested where $E[V \mid r], E[N \mid r], E[r \mid V], E\left[V^{\prime} \mid N^{\prime}\right], E\left[N^{\prime} \mid V^{\prime}\right], E[V / N \mid r]$, and empirical finding and theory matched very well.

${ }^{31}$ So looking at the fund turnover will not be the right thing to do. To provide liquidity, or avoid looking like a "closet indexer" a large fund may maintain a fairly large turnover, though the "price moving" trades will indeed happen less often, as they move prices a lot.

${ }^{32}$ The above bullets make respectively $2,2,5 \cdot 3=15,1$ and 4 predictions.

${ }^{33}$ The printout of the 56 theoretical relationships $E[Y \mid X]$ involving $V, N, V^{\prime}, N^{\prime}, r, N^{\prime} / N, r^{2}, V / N$ is available from the authors upon request.
} 

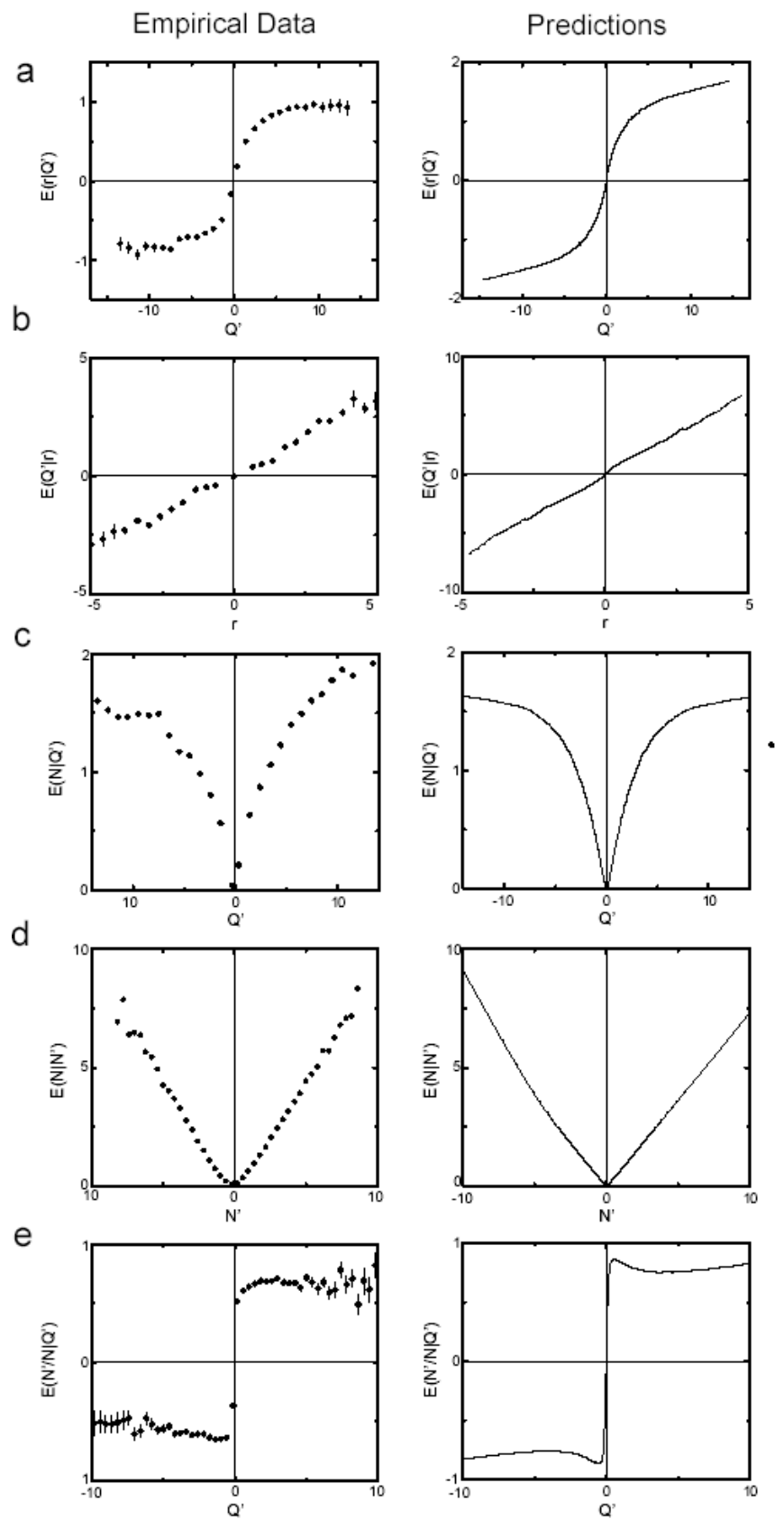

Figure 8: Conditional expectations for (a) $E\left[r \mid Q^{\prime}\right]$, (b) $E\left[Q^{\prime} \mid r\right]$, (c) $E\left[N \mid Q^{\prime}\right]$, (d) $E\left[N \mid N^{\prime}\right]$, and (e) $E\left[N^{\prime} / N \mid Q^{\prime}\right]$. We form, for each interval $\Delta t=15 \mathrm{~min}$, the quantities (i) $r$, the returns, (ii) $Q_{B}$ (resp. $Q_{S}$ ), the number of shares exchanged in a buyer (resp. seller) initiated trade, (iii) $N_{B}$ (resp. $N_{S}$ ), the number of buyer (resp. seller) initiated trades, $Q^{\prime} \equiv Q_{B}-Q_{S}$, and $N^{\prime} \equiv N_{B}-N_{S}$. The left panel shows the empirical values for the 116 frequently traded stocks in the Trades and Quotes database for 1994-1995. This represents 1.5 million observations. Variables are normalized to unit variance after setting the mean to zero; for variables such as volume for which the variance is divergent, we have by normalized the first moment instead. The right panel shows the model's predictions, which agree well with the empirical data. 


\subsubsection{The public news based (efficient markets) model}

In this model, price movements reflect without frictions public pieces of news. One would need to make large number of assumptions to make this model fit the empirical power laws. First, because returns reflects news, we have to assume that the distribution of news has $\zeta_{r}=3$.

This is not the only difficulty. In the benchmark where volumes do not move prices, there is no reason for big traders to trade less than small ones. With no price impact, essentially all models that traders should trade in amount proportional to their sizes, so that the volume $V_{i}$ traded by trader $i$ will be equal to $V_{i}=a_{i} S_{i}$ for $a_{i}$ a random variable that do not scale with size. Then, we would get $\zeta_{V}=\zeta_{S}=1$.This prediction is a major difficulty with the news approach. Otherwise, to work with the public news model, one would have to assume $\zeta_{V}=3 / 2$, for some inexplicable reason. Hence the values of $\zeta_{r}$ and of $\zeta_{Q}$ are just assumed in that model and do not arise as a consequence of a theory.

\subsubsection{A mechanical "price reaction to trades" model}

In this model, the desired $N$ trades are i.i.d. The price has a reaction $f\left(\varepsilon_{j} q_{j}\right)$ to each trade of size $q_{j}$ and $\operatorname{sign} \varepsilon_{j}$. So $r=\sum_{j=1}^{J} f\left(\varepsilon_{j} q_{j}\right)$ and the other variables are defined as in (49)-(52).

This model is a variant of our model, and so it will tend to generate qualitatively right $E[Y \mid X]$ curves. However there are problems with this model. Firstly, we need to assume $\zeta_{Q}=3 / 2$. However, given $\zeta_{S}=1$, the natural prediction would be $\zeta_{Q}=1$. Secondly, we need to assume $\zeta_{N} \simeq 3$. It

could be any other value. Thirdly, we also need to assume a price impact $\Delta p \approx V^{1 / 2}$ to get the shape of $E\left[r^{2} \mid Q\right]$.

\subsubsection{Random bilateral matching}

This has been proposed by Solomon and Richmond (2001). The model says that two traders of size $S, S^{\prime}$ meet, and the resulting volume is $\max \left(S, S^{\prime}\right)$ and the price impact is $\min \left(S, S^{\prime}\right)$. While this approach is reasonable, it is not without problems. As the initial size distribution is $\zeta_{S}=1$, $\zeta_{\min \left(S, S^{\prime}\right)}=2$, so that one would predict $\zeta_{Q}=\zeta_{r}=2$. The authors rely on a scaling exponent of the size of agent $\zeta_{S}=3 / 2$, as is approximately the case in the US and the UK in the late 1990s, but not in other periods. A major difficulty for this theory is that empirically the exponent is individual wealth is highly variable. Feenberg and Poterba (1993) show that the exponent for the wealth of U.S. individuals had large variations from 1.5 to 2.5 in the last 30 years. This large variability is confirmed in a series of papers on U.S. and international data by Piketty and Saez (e.g. Piketty and Saez 2002).

\subsection{Related empirical findings}

\subsubsection{Other models for returns}

The empirical literature has proposed other distributions. We are more confident about our findings rely on much larger number of data points, hence quantify the tails more reliably. We can also explain previous findings in light of ours. Andersen et al. (2001) show that the bulk of the distribution of realized volatility is lognormal. In independent work Liu et al. (1999) show that while this is true, the tails are power law. Gopikrishnan et al. (1999) also report how, with a small sample that looks only at the center rather than the tails of the distributions, one would find Lévy or truncated Lévy distributions, as was found by Mantegna and Stanley (1995).

Finally Ané and Geman (2000) propose a model which is in essence $r_{t}=\sum_{j=1}^{N_{t}} u_{i}$ where $u_{i}$ are normal shocks. This is a variant of the Clark (1973) model where the clock is driven by the number 
of trades rather than the volume. This model has an important empirical problem: from $\zeta_{N} \geq 3$, as $|r| \sim N^{1 / 2}$ in this model, we conclude $\zeta_{r}=2 \zeta_{N} \geq 6$, so that model fails to reproduce the empirical cubic law of returns. In fact, in our model, results similar to Ané-Geman would be found: for instance, $E\left[r^{2} \mid N\right]$ looks almost linear (though it is not really linear). Under the null of our model, their model is a good, but ultimately inaccurate, representation of reality.

\subsubsection{Buy / Sell asymmetry}

Our basic model has built-in full symmetry between positive and negative tails. This is not a bad approximation of reality: the exponents are roughly identical, around 3. However, it is true that the exponents are slightly lower (fatter tails) in the negative tail that in the positive one. More generally, one finds a bit more skewness in the negative tail. One reason may found in the "leverage effects". Volatility goes up after negative returns. Mechanically, this skews to the left the returns over a non-infinitesimal $\Delta t$, and the effect grows with $\Delta t$.

Our framework suggests another hypothesis. Suppose the frequency of arrival $f$ of liquidity providers is higher for desired sells (so the liquidity providers have to buy the asset) than buys. Chan and Lakonishok (1993) report the "Street" explanation for this ${ }^{34}$. When one looks to buy a large amount, one can only deal with liquidity providers who already own the assets, if shorting is difficult. But all liquidity provider can absorb a sell order. So it is easier to meet counterparts for sells than for buys, i.e. $f_{+}<f_{-}$, where $f_{+}$(resp. $f_{-}$) is frequency of arrival of liquidity providers that are willing to be counterpart of a buy (resp. sell) order, $f_{-}$. By plugging $f_{+}$or $f_{-}$in (70) we see that $\left|\Delta p_{+}\right|>\left|\Delta p_{-}\right|$, i.e. the theory predicts higher price movements for buys than for sells, though also identical exponents.

\subsection{Link with the behavioral and excess volatility literature}

There is a growing literature suggesting that many movements in the markets are hard to explain by any kinds of news. Cutler, Poterba, Summers (1989) look at daily returns, Fair (2002) looks at the largest 1 to $5 \mathrm{~min}$ S\&P500 returns. Both studies find that many are not explainable by news. The puzzle is stated in related ways by French and Roll (1986), Roll (1988), and Shiller (1989). Trading per se seems to create volatility (Evans and Lyons 2002), and returns, even ex post, are hard to explain. Our theory would predict that, on those events of large movements with no news, trading volume is abnormally high. It would also predict that those returns should mean revert.

\subsection{Link with the microstructure literature}

The literature from which this paper draws most is perhaps the microstructure literature. This literature is extremely vast (for reviews see e.g. O'Hara 1997 and Biais et al. 2002), so we will only mention the most directly relevant pointers. Our theory makes predictions above the relationships between volume and price impact, and we reviewed in Section 4 the papers directly pertaining to our theory. Other relevant papers, that show a long run impact of trading on prices include Evans and Lyons (2002), and the analysis of volume in Wang (1994), Lo and Wang (2001). Rather than the fine analysis proposed in the literature (e.g. Madhavan and Cheng 1997), we adopted a fairly stylized view of trading institutions. This was motivated by the fact that the cubic exponents arise for different market structures, so that our explanation should not depend too finely on a specific market structure.

Our goal is to explain the cubic power laws, and the patterns in trading activity at a fairly high degree of aggregation (1/2 hour as opposed to trade by trade). Hence we highlight some

\footnotetext{
${ }^{34}$ Saar (2001) provides an alternative explanation based on asymmetric information.
} 
traits of reality - mainly the heterogeneity between the size of agents, and the trade-off between execution cost and execution time - that are typically not central to the microstructure literature. Another feature is that we are agnostic about the importance of information asymmetry in the determination of asset prices. We highlight more "macro" phenomena that arise from aggregation, rather the detailed understanding of micro situations that tend to be the focus of the microstructure literature. Hence, our model and the models of this literature appear quite different. Still, it would be desirable, in further research, to merge the rich informational and institutional understanding from the microstructure literature and the more macro approach of the present paper.

\subsection{Link with the "econophysics" literature}

This paper is part of a broader movement utilizing tools from physics in for the study of economic issues. In the same way that the application of physics to earth sciences and biology are called geophysics and biophysics, this literature is sometimes called "econophysics". This literature was pioneered by Mandelbrot (1963). This literature is growing, and includes Bak, Chen, Scheinkman and Woodford (1993), Bouchaud and Potters (2000), Canning et al. (1998), Gabaix (1999, 2003), Plerou et al. (1999), Lux and Sornette (2002), Lévy, Lévy and Solomon (2000), Mantegna and Stanley (2000).

\section{Conclusion}

This paper proposed a theory of power law behavior of several variables that describe financial market activity. It explains the cubic and half-cubic exponent of returns and volume, and also makes many predictions about other quantities. Section 5.2 presents numerical predictions about 24 other power law exponents. This offers ample room for future research. We have examined some of its predictions, in particular in Figure 8. Further research ${ }^{35}$ is made is by the modular structure of our arguments, as shown in by Figure 6 .

This theory can also be used for policy analysis. The cubic law predicts the existence of very large returns, and Gabaix et al. (2003c) report that stock market crashes such as the 1929 or 1987 crashes do do not seem to be outliers to the cubic law. Hence if we understand the origin of the cubic law, we may understand the origin of stock market crashes. Within the present framework, Gabaix et al. (2003d) examine the performance of traditional proposals against crashes, such as the Tobin tax or circuit breakers. It shows that they not dampen crashes, and works out different proposals that in principle would achieve that goal.

On the substantive front, our model provides one possible quantitative theory of excess volatility in asset markets: it is simply due to the desire to trade of large traders (perhaps stimulated by news). More precisely, it is the power law 1 of the distribution of sizes that generates, through an intelligent though not hyperrational (people trade too much) trading process, the power laws of 3 in returns and $3 / 2$ in volume. On the methodological front, we introduce some new questions that finance theories should answer. Matching, as we do, the quantitative empirical regularities established here (in particular explaining the exponents of 3 and $3 / 2$, not merely assuming them), in particular the cubic laws, would be a sine qua non criterion for the admissibility of a of volume and volatility. We hope that the regularities we established will sharply constrain, and guide, future theories. Given

\footnotetext{
${ }^{35}$ Our model has very little to say about the low frequency structure of trading. Our model contains a series of independent trading decisions, and is silent about the time-correlations in market activity. It is straightforward to propose simple extensions of it, such as an GARCH-type model for the propensity to trade $J_{t}$, so as to qualitatively account for the well-known long term memory in volatility (where $J_{t+1}$ depends positively on past $J_{t}^{\prime} s$ and $\left|r_{t}\right|$ ). The proper analysis of this, however, is outside the scope of this paper. We are investigating this in ongoing research.
} 
its empirical success and its simple structure, the present model might a useful point of departure to think about those issues. 


\section{Appendix A: Some power law mathematics}

We present here some basic facts about power law mathematics, and show how their great aggregation properties makes them especially interesting for both theoretical and empirical work. They also show how our predictions are robust to other sources of noise.

A random variable $X$ has power law behavior if there is a $\zeta_{X}>0$ such that:

$$
P(X>x) \sim \frac{1}{x^{\zeta X}}
$$

so that the probability density $p(x)$ follows:

$$
p(x) \sim \frac{1}{x^{\zeta x+1}}
$$

A more general definition is that there is a "slowly varying" 36 function $L(x)$ and a $\zeta_{X}$ s.t. $p(x) \sim L(x) / x^{\zeta x+1}$, so that the tail follows a power law "up to logarithmic" (by some abuse of language) corrections.

$\zeta_{X}<\zeta_{Y}$ means that $X$ has fatter tails than $Y$, hence the large $X^{\prime} s$ are (infinitely, at the limit) more frequent than large $Y^{\prime} s$.

The definition implies that with $\alpha>0, E\left[|X|^{\alpha}\right]=\infty$ for $\alpha>\zeta_{X}$, and $E\left[|X|^{\alpha}\right]<\infty$ for $\alpha<\zeta_{X}$. As an example, if for returns $\zeta_{r}=3$, then $E\left[|r|^{\alpha}\right]$ for $\alpha>3$. In particular, the kurtosis of returns in infinite ${ }^{37}$, and their skewness borderline infinite. For $Y$ a normal, lognormal, exponential, the formal power law exponent is $\zeta_{Y}=\infty$, given $Y$ dies out faster than any power laws.

Power laws have great aggregation properties. The property of being power law is conserved under addition, multiplication, polynomial transformation, min, max. The motto is that, when we combine two power law variables, "the biggest (fattest=smallest exponent) power law dominates". Indeed, for $X, Y$ independent variables, we have the formulaire:

$$
\begin{aligned}
\zeta_{X+Y} & =\zeta_{X \cdot Y}=\zeta_{\max (X, Y)}=\min \left(\zeta_{X}, \zeta_{Y}\right) \\
& =\text { power law of fattest variable } \\
\zeta_{\min (X, Y)} & =\zeta_{X}+\zeta_{Y}
\end{aligned}
$$

For instance, if $X$ is a power law for $\zeta_{X}<\infty$, and $Y$ is power law variable with an exponent $\zeta_{Y} \geq \zeta_{X}$, or even normal, lognormal or exponential variable (so that $\zeta_{Y}=\infty$ ), then $X+Y, X \cdot Y$, $\max (X, Y)$ are still power laws with the same exponent $\zeta_{X}$. So multiplying by normal variables, adding non fat tail noise, summing over i.i.d. variables preserves the exponent. So (i) this makes theorizing with power law very streamlined; (ii) this lets the empiricist hope that those power laws can be measured, even if there is a fair amount of noise in the data. One does not need to carry around the additional noise, because though it will affect variances etc., it will not affect the power

${ }^{36} L(x)$ is said to be slowly varying (e.g. Embrechts et al. 1997, p.564) if

$$
\lim _{x \rightarrow \infty} L(t x) / L(x)=1 \text { for all } t>0 .
$$

Prototypical examples are $L=a$ and $L(x)=a \ln x$ for a non-zero constant $a$.

${ }^{37}$ This makes the use of the kurtosis invalid. As the theoretical kurtosis is infinite, empirical measures of it are essentially meaningless. As a symptom, according Lévy's theorem, the median sample kurtosis of $T$ i.i.d. demeaned variables $r_{1}, \ldots, r_{T}$, with $\kappa_{T}=\left(\sum_{i=1}^{T} r_{i}^{4} / T\right) /\left(\sum_{i=1}^{T} r_{i}^{2} / T\right)^{2}$, increases to $+\infty$ like $T^{1 / 3}$, as the sample size $T$ increases. The use of kurtosis should banished. As a simple diagnostic for having "fatter tail than from normality", we would recommend, rather than the kurtosis, quantile measures such as $P\left(\left|(r-\langle r\rangle) / \sigma_{r}\right|>1.96\right) / .05-1$, which is positive if tails are fatter than a normal. 
law exponent. Power law exponents carry over the "essence" of the phenomenon: smaller order effects do not affect the power law exponent.

For instance, say a theory, for instance ours, gives a mechanism for $R$, with $\zeta_{R}=3$. Other things are going on, so that in reality, we observe:

$$
\widetilde{r_{i t}}=\widetilde{a_{i t}} \widetilde{R}_{i t}+\widetilde{b_{i t}}
$$

For instance, the liquidity of the market varies, so that $\widetilde{a_{i t}}$ is random, and news can affect prices $\widetilde{b_{i t}}$ without affecting volume. But even then, we will have $\zeta_{r}=\zeta_{R}=3$ if $\widetilde{a}, \widetilde{b}$ are smaller order effects, i.e. have thinner power laws $\left(\zeta_{a}, \zeta_{b} \geq 3\right)$. If the theory of $R_{i t}$ capture the first order effects (i.e. those with dominating power law), its predictions for the power law exponents of the "noised up" empirical counterpart $\widetilde{r_{i t}}$ will still be true.

Proof. See Breiman (1965) and Gnedenko and Kolmogorov (1968) for rigorous proofs, and Sornette (2000) for intuitive, heuristic derivations. Here we just indicate the proofs for the min and max operations. For $\zeta_{\min (X, Y)}$, we have:

$$
\begin{aligned}
P(\min (X, Y)>x) & =P(X>x \text { and } Y>x)=P(X>x) P(Y>x) \\
& =\frac{k}{x^{\zeta X}} \frac{k^{\prime}}{x^{\zeta y}}=\frac{k k^{\prime}}{x^{\zeta X+\zeta_{Y}}}
\end{aligned}
$$

Also,

$$
\begin{aligned}
P(\max (X, Y)>x) & =1-P(\max (X, Y)<x)=1-P(X<x \text { and } Y<x) \\
& =1-P(X<x) P(Y<x) \\
& =1-\left(1-\frac{k}{x^{\zeta X}}\right)\left(1-\frac{k^{\prime}}{x^{\zeta y}}\right) \sim \frac{k^{\prime \prime}}{x^{\min \left(\zeta_{X}, \zeta_{Y}\right)}}
\end{aligned}
$$

where $k^{\prime \prime}=k$ if $\zeta_{X}<\zeta_{Y}, k^{\prime \prime}=k^{\prime}$ if $\zeta_{X}>\zeta_{Y}$, and $k=k+k^{\prime}$ if $\zeta_{X}=\zeta_{Y}$.

This generalizes to $K$ independent power law variables:

$$
\begin{aligned}
\zeta_{X_{1}+\ldots+X_{K}} & =\zeta_{X_{1} \ldots . X_{K}}=\zeta_{\max \left(X_{1}, \ldots, X_{K}\right)}=\min \left(\zeta_{X_{1}}, \ldots, \zeta_{X_{K}}\right) \\
\zeta_{\min \left(X_{1}, \ldots, X_{K}\right)} & =\zeta_{X_{1}}+\ldots \zeta_{X_{K}}
\end{aligned}
$$

Finally, a useful formula is:

$$
\zeta_{X^{\alpha}}=\frac{\zeta_{X}}{\alpha} \text { for } \alpha>0
$$

The proof is the same as in Proposition 2.

\section{Appendix B: Generalization of Theorem 3 to non i.i.d. set- tings}

We sketch here how our main results, including Theorem 3, extend without difficulty to non i.i.d. settings. For instance, there can be autocorrelation in market liquidity and trade arrival. Also, different traders can have different trading frequencies. We index large traders by $a$. We say that $\tau_{a t}=1$ if $a$ does trade at time $t$ in that interval, $\tau_{a t}=0$ otherwise. So, the total volume and returns 
created by the fund are respectively:

$$
\begin{aligned}
& V_{a t}=\tau_{a t} k_{a t} S^{\delta_{a}} \\
& r_{a t}= \pm \tau_{a t} \lambda_{a t} V_{a t}^{1 / 2}
\end{aligned}
$$

for some possibly time-varying, correlated prefactors $k_{a t}, \lambda_{a t}$. While the paper is, for simplicity, i.i.d., this formulation allows the prefactors $k_{a t}, \lambda_{a t}$ and other prefactors to be correlated between stocks and across time. For instance, a period with high $k_{a t}$ and low $\lambda_{a t}$ has large typical volume and low price impact. This captures the presence of time-varying liquidity and volume that have been detected in empirical data (see e.g. Lobato and Velasco 2000).

The aggregate volume and return are:

$$
\begin{aligned}
Q_{t}^{A g g} & =\sum_{a} V_{a t} \\
r_{t}^{A g g} & =\sum_{a} r_{a t}
\end{aligned}
$$

as each trader creates a volumes $V_{a t}$ and moves prices by an amount $r_{a t}$. They correspond to the observed return and volume, simply called $r_{t}$ and $V_{t}$ in the paper.

We need to show that $\zeta_{r}{ }^{A g g}=3$ and $\zeta_{Q^{A g g}}=3 / 2$ in that context also. Showing this is a bit involved. The difficult part is to show that $r_{a t}$ and $V_{a t}$ have respectively power law 3 and 1.5. The general properties of power laws make $r_{t}^{A g g}$ and $Q_{t}^{A g g}$ inherit the power laws of $r_{a t}$ and $V_{a t}$, i.e. $\zeta_{r^{A g g}}=2 \zeta_{Q^{a g g}}=3$.

We now proceed to show $r_{a t}$ and $V_{a t}$ have respectively power law 3 and 1.5. The result is stronger than the one in the body of this paper:

Proposition 11 We index funds by a, and dates by $t$. We call $\theta_{a}$ the "trading style" of fund a, and $\varepsilon_{a t}$ indexes market-wide and fund-specific time-varying characteristics. We assume (i) fund's sizes follow Zipf's law; (ii) the price impact follows

$$
\left|r_{a t}\right|=\lambda_{a t} V_{a t}^{1 / 2}
$$

(iii) fund $a$, of size $S_{a}$, trades in volumes

$$
V_{a t}=k_{a t} S^{\delta_{a}}
$$

(iv) fund a's total transaction costs are equal to $C_{a} S_{a}$. We assume the following stochastic structure:

$$
\begin{aligned}
\lambda_{a t} & =\lambda\left(\theta_{a}, \varepsilon_{a t}\right) \\
k_{a t} & =k\left(\theta_{a}, \varepsilon_{a t}\right) \\
\delta_{a} & =\delta\left(\theta_{a}\right) \\
C_{a} & =C\left(\theta_{a}\right)
\end{aligned}
$$

for some functions $\lambda, k, \delta$ and $C$ with compact support in $(0, \infty)$, and $\theta_{a}, \varepsilon_{a t}$ are (possibly multidimensional) random variables, independent of the sizes $\left(S_{a}\right)_{a=1 \ldots n}$, identically distributed across a. $\left(\theta_{a}\right)_{a=1 . . n}$ and $\left(\varepsilon_{a t}\right)_{t ; a=1 \ldots, n}$ are independent, but the $\varepsilon_{a t}$, assumed to be stationary, can be correlated across $t$ 's and $a$ 's. The probability that fund a will trade at $t$ has the form

$$
F_{a t}=G\left(S_{a}, \theta_{a}\right) H\left(\theta_{a}, \varepsilon_{a t}\right)
$$


so that it is dependent on shocks $\varepsilon_{a t}$ and the size $S_{a}$ but in a form that is weakly separable. Then, the distribution of the individual (before aggregation) volumes and returns follow power law distributions with exponents:

$$
\zeta_{Q_{a t}}=3 / 2 \text { and } \zeta_{r_{a t}}=3
$$

We comment on the hypotheses. There are four main improvements upon the text, which assumes constant functions $\lambda, k, \delta$ and $C$, and $F_{a t}=G\left(S_{a}\right)$.

Firstly, $\delta_{a}$ and $C_{a}$ need not be the same across funds: they can depend on the "trading style" $\theta_{a}$ of fund $a$, though we keep the assumption that they are independent of the size $S_{a}$. Secondly, the probability of trading, $F_{a t}$, the volume traded $V_{a t}$, and the price impact $\left|r_{a t}\right|$ can vary from trade to trade. This can reflect changing "market conditions," such as the liquidity $1 / \lambda$. For instance, the probability of trading $F_{a t}$ could increase with the probability of trading. Thirdly, we allow intertemporal correlation in the noise structure via $\varepsilon_{a t}$. This allows for autocorrelation in the trading frequency. Fourthly, the probability of trading $F_{a t}$, the volume traded $V_{a t}=S^{\delta_{a}} k\left(\theta_{a}, \varepsilon_{a t}\right)$, and the price impact, $\left|r_{a t}\right|=\lambda\left(\theta_{a}, \varepsilon_{a t}\right) V_{a t}^{1 / 2}$ can be correlated. For instance, the volume $k$ could be higher when the liquidity $1 / \lambda$ is higher. The fact that $\varepsilon_{a t}$ is multidimensional allows a large class of imperfect correlations: one component of $\varepsilon_{a t}$ can be a market-wide term dependent on $t$, and another can be an idiosyncratic term dependent on $a$ and $t$. Also, the price impact can vary on the "nature" of the fund, represented by $\theta_{a}$ (for instance degree of impatience represented by $\mu$ in the paper can be part of a multidimensional $\theta_{a}$ ).

The crucial hypothesis is that the distributions of $\left(\delta_{a}, k_{a t}, \lambda_{a t}, C_{a}\right)$ are "scale invariant," i.e. do not depend on $S_{a}$. The essence of the proof is similar to the one in the paper, though the notations are more involved. The proof is fairly technical, and would be too long to reproduce here. It is available from the authors upon request.

\section{Appendix C: The model with general trading exponents}

We present here the general structure of the model. It allows to see which assumptions are crucial to get the "cubic" exponents. Also, natural quantities, the "exponents of trading", emerge. Their direct measurement is an interesting task for future research. We call $\zeta_{S}$ and $\zeta_{L}$ the Pareto exponents of the large traders and the liquidity providers, respectively. We make the following Ansatz for the behavior of our variables in the large $S,|\Delta p|$, and $T$ limit:

- A large trader of size $S$ will make large position of size $V=S^{\delta}$ at an annual frequency $S^{-\phi}$.

- If a large traders makes an order at a price concession $\Delta p$, then the frequency of arrival of liquidity traders is $f=\Delta p^{\alpha}$, and each liquidity provider of size $s$ supplies a number of shares $q=s \cdot \Delta p^{\beta}$.

- A trader of size $S$ will adjust the trading frequency so as to pay a proportional amount of transactions costs $S^{\kappa}$.

- The large trader, in a given trade of size $V$, wants to minimize a loss function $\Delta p+\mu T^{\tau}$ where $T$ is the mean time needed to find enough liquidity providers to complete the trade. For instance $\tau<1$ would correspond to a concave arrival time of information to the market.

The original model corresponds to a value of 0 for $\alpha$ and $\kappa$, and a value of 1 for $\zeta_{S}, \zeta_{L}, \beta$, and $\tau$. The next Proposition describes the results. 
Proposition 12 Under the above conditions, which generalize the model in the text, the exponents of returns, volumes, individual trades, and number of trades are:

$$
\begin{aligned}
\zeta_{r} & =\frac{\zeta_{S}+\phi}{\delta}\left(\frac{1}{\tau}+\alpha+\beta\right) \\
\zeta_{Q} & =\frac{\zeta_{S}+\phi}{\delta} \\
\zeta_{q} & =\zeta_{Q}+\left(\zeta_{L}-1\right) \frac{\alpha \tau+1}{(\alpha+\beta) \tau+1} \\
\zeta_{N} & =\frac{\zeta_{S}+\phi}{\delta}\left(1+\frac{\beta \tau}{\alpha \tau+1}\right)
\end{aligned}
$$

The measured price impact function is $\Delta p \sim V^{\gamma}$ with

$$
\gamma=\frac{\tau}{(\alpha+\beta) \tau+1}
$$

and the exponent of the trading frequency is:

$$
\phi=\delta(1+\gamma)-\kappa-1
$$

Hence the exponents of returns, volume and number of trades increase with the exponent of the distribution of traders $\zeta_{S}$. Somehow forcing large traders to trade less, other things equal, is going to decrease the fat-tailness of returns, i.e. increase $\zeta_{r}$. Decreasing the time pressure to trade, in the sense of lowering $\tau$, also decreases the fat-tailness of volumes. Equation (65) shows how to get values for $\gamma$ different from $1 / 2$, the value we mostly used in the paper.

Proof. The probability that a target volume $V$ is $>x$ is:

$$
P(V>x) \sim \int_{S^{\delta}>x} S^{-1-\zeta_{S}} S^{-\phi} d S \sim x^{-\frac{\zeta_{S}+\phi}{\delta}}
$$

so that the distribution of target volumes is (62).

After a time $t$, the large trader has received on average $\sim \Delta p^{\alpha+\beta} t$ shares. So the mean time needed to realize a trade of size $V$ is $T=h^{\prime} V / \Delta p^{\alpha+\beta}$, and the objective function is:

$$
\min _{\Delta p} \Delta p+\mu h^{\prime} \frac{V^{\tau}}{\Delta p^{\tau(\alpha+\beta)}}
$$

which yields $\Delta p \sim V^{\gamma}$ with $\gamma$ given by (65). This gives (61) by (56).

Each transaction costs $V \Delta p(V) \sim V^{1+\gamma} \sim S^{\delta(\gamma+1)}$, so the annual amount will be $\sim$

$$
S^{-\phi} \cdot S^{\delta(1+\gamma)}=S^{1+\kappa}
$$

which gives (66).

The number of trades is $n \sim V / \Delta p^{\beta}$, which gives

$$
n \sim V^{\frac{\alpha \tau+1}{(\alpha+\beta) \tau+1}} .
$$

and (64) by (56). 
An individual trade $>x$ involves a target volume $V>x$, and a fraction equal to $P\left(s_{i} \Delta p^{\beta}>x\right)$ of the $n(V) \sim V / \Delta p^{\beta}$ liquidity traders who meet his demand. So:

$$
\begin{aligned}
P\left(q_{i}>x\right) & \sim \int_{V>x} V^{-1-\zeta_{Q}} \cdot n(V) d V \cdot \int_{s_{i} \Delta p(V)^{\beta}>x} s_{i}^{-1-\zeta_{L}} d s_{i} \\
& \sim \int_{V>x} V^{-1-\zeta_{V}} \frac{V}{V^{\beta \gamma}}\left(\frac{V^{\beta \cdot \gamma}}{x}\right)^{\zeta_{L}} d V \\
& =x^{-\zeta_{L}} \int_{V>x} V^{-\zeta_{Q}-\beta \gamma+\zeta_{L} \cdot \beta \cdot \gamma} \\
& \sim^{-\zeta_{Q}-\left(\zeta_{L}-1\right)(1-\beta \gamma)}
\end{aligned}
$$

so that $\zeta_{q}=\zeta_{Q}+\left(\zeta_{L}-1\right)(1-\beta \gamma)$, which gives $(63)$.

\section{Appendix D: A model for the linear supply function (38)}

We present a possible model equation (38), where a liquidity provider of size $s_{i}$ gives $q_{i}$ for a price concession $\Delta p$. As we try to keep the theory modular, the sub-model is essentially independent of the rest of the theory. Our model is a simple version of the inventory cost models of e.g. Grossman and Miller (1988) and Stoll (1978). For simplicity, we drop the subscript $i$ in this Appendix. We use a very stark model to side-step the complexities identified by O'Hara (1995).

The liquidity provider has a net assets $s$, and a mean variance objective function on his returns at some horizon ${ }^{38} \mathrm{H}$, with a zero riskless rate.

$$
U=E\left[W_{H}\right]-\frac{\rho}{2 s} \operatorname{var} W_{H}
$$

The factor $\rho / s$ ensure that the risk taking behavior is independent of wealth, as $U$ is homogenous of degree 1 in $\left(W_{H}, s\right)$. If he holds $A$ shares, with expected return $m$ and volatility $\sigma$, his utility is:

$$
U^{*}=W+H\left(m A-\frac{\rho}{2 s} \sigma^{2} A^{2}\right)
$$

We will assume that he is allowed to supply the liquidity once before he can sell it again, costlessly, to the market. On average it takes a time $L$ to do this. We assume that he cannot rebalance his portfolio in the meantime. Formally, we could model $L$ as the average time before some other large liquidity provider arrives, who would absorb liquidity costlessly. It could also be the amount of time to sell the block at essentially no cost.

At the optimum, the liquidity provide holds $A^{*}$ share of the asset. By optimization of (68)

$$
A^{*}=\frac{m s}{\rho \sigma^{2}} \text {. }
$$

If he supplies $q$ shares to the trader, he will hold $A^{*}-q$ shares between times 0 and $L$ and the optimum quantity, $A^{*}$, between times $L$ and $H$. He pockets the price concession $q \Delta p$. So his utility is:

$$
U^{* *}=W+q \Delta p+L\left[m\left(A^{*}-q\right)-\frac{\rho}{2 s} \sigma^{2} H\left(A^{*}-q\right)^{2}\right]+(H-L)\left[m A^{*}-\frac{\rho}{2 s} \sigma^{2} A^{* 2}\right]
$$

\footnotetext{
${ }^{38}$ Formally, this could be a Poisson time at which his portfolio is evaluated.
} 
We assume that the large trader has full bargaining power, so that the liquidity provider must be just indifferent between supplying the shares or keeping them. This happens if $U^{* *}=U^{*}$, i.e.

$$
\Delta p=\rho \sigma^{2} L q / s
$$

So we get the supply function (38) with

$$
\alpha=\frac{1}{\rho \sigma^{2} L}
$$

This means that the liquidity, $\alpha$, is higher when assets have low volatility $\sigma$, short resell time $L$, and when the effective risk aversion $\rho$ of the liquidity providers is low. Those are the traditional features of inventory models.

The argument above holds much more generally. Costs of a suboptimal holdings are second order, hence equal to $\Lambda q^{2}$ for some constant $\Lambda$. The excess cash pocketed by the liquidity provider, $q \Delta p$, has to compensate that, so that: $q \Delta p=\Lambda q^{2}$, and $\Delta p=\Lambda q$. The form $\Delta p=\lambda s q$ comes from the scale-invariance in $s$.

\section{Appendix E: Algorithm for the simulations}

In the period $\Delta t$ considered, there will be $J$ such "trading rounds" where a big trader creates one or more trades. Each round $j$ has a sign $\varepsilon_{j}= \pm 1$, and gives rise to $r_{j}, V_{j}, n_{j}, V_{j}^{\prime}$, and $n_{j}^{\prime}$. We define:

$$
\begin{aligned}
r & =\sum_{j=1}^{J} r_{j}=\sum_{j=1}^{J} \varepsilon_{j} V_{j}^{1 / 2} \\
Q & =\sum_{j=1}^{J} V_{j}, N=\sum_{j=1}^{J} n_{j} \\
Q^{\prime} & =\sum_{j=1}^{J} \varepsilon_{j} V_{j}, N^{\prime}=\sum_{j=1}^{J} \varepsilon_{j} n_{j}
\end{aligned}
$$

We have $E\left[n_{j} \mid V_{j}\right]=V_{j}^{1 / 2}$ up to numerical prefactors. In reality, empirical returns the $r$ above, plus some noise due e.g. to news and other market events outside the model. However, those extraneous events do not affect the large events and do not affect our graphs in major ways.

One simulates $T$ time intervals indexed by $t=1, \ldots, T$ in the following way.

1. One draws an integer value $J_{t}$ drawn at random, as the integer part of $10 e^{g_{t}}$, with $g_{t}$ a standard normal.

2. One draws $v_{t}=e^{.5 u_{t}}$ and $\eta_{t}=e^{.5 v_{t}}$ with $u, v$ i.i.d. standard normals. For $j=1 \ldots J_{t}$, one draws $Q_{j}$ with $\zeta_{Q}=3 / 2$, and $\varepsilon_{j}= \pm 1$ with probability $1 / 2$, and sets:

$$
\begin{aligned}
r_{j} & =\varepsilon_{j} \nu_{t} \eta_{t}^{-1} Q_{j}^{1 / 2} \\
N_{j} & =\nu_{t} \eta_{t} Q_{j}^{1 / 2} \\
N_{j}^{\prime} & =\varepsilon_{j} N_{j}
\end{aligned}
$$

Note that $\nu_{t}, \eta_{t}$ are constant across the $J_{t}$ rounds $j$ of the time interval.

3. Applying (70), one gets a five values $\left(r, Q, N, Q^{\prime}, N^{\prime}\right)$ which are stored as $\left(r_{t}, Q_{t}, N_{t}, Q_{t}^{\prime}, N_{t}^{\prime}\right)$. 
One calculated the various $E[Y \mid X]$ graphs on the data set made up of those values $T$ vectors $\left(r_{t}, Q_{t}, N_{t}, Q_{t}^{\prime}, N_{t}^{\prime}\right)$. To facilitate comparisons across stocks, one rescales the variables - demean them, and divide them by their standard deviation if this standard deviation exists ${ }^{39}$, otherwise by their absolute mean.

Equations (71) and (72) are intended to be the analogues to the theoretical (44) and (46) resp. $r$ and $N$ vary like $Q^{1 / 2}$, but there are varying "market conditions" $\nu_{t}=(\alpha \bar{s})^{-1 / 2}$ (a measure of the "depth" of each liquidity provider of the market at the given point in time), and $\eta_{t}=\mu^{1 / 2}$ (the arrival rate of liquidity providers). While those quantities must have some randomness, we do not have strong prior on the extent of their randomness, and any way they play only a minor role in the model. So, largely arbitrarily, we chose make them equal to $e^{.5 u}$, with $u$ a standard normal. The .5 factor captures a relatively small randomness, but the results are largely insensitive to that choice. Finally, the value of $J$ around 10 was so that the average number of trades in a given half hour match roughly the empirical one, without further effort to calibrate the model..

\section{Appendix F: Confidence intervals and tests when a vari- able has infinite variance}

\subsection{Construction of the confidence intervals for Figure 7}

In a given bin conditioned by $Q=Q_{i}$, with $k$ elements $r_{1}^{2}, \ldots, r_{k}^{2}$, the point estimate of $E\left[r^{2} \mid Q=Q_{i}\right]$ is naturally the sample mean of the $r_{j}^{2}$, which we call $m$. Getting a confidence interval for $m$ is delicate, as $r^{2}$ has infinite variance, so the standard approach relying on asymptotic normality is invalid. But the theory of self-normalizing sums of Logan et al. (1973) shows that if $\mu$ is the true mean and $\sigma_{r^{2}}$ is empirical standard deviation of the $r_{j}^{2}$ in the bin, then Student's $t=k^{1 / 2}(m-\mu) / \sigma$ tends to a non-Gaussian distribution that depends only on the tail exponent of $r^{2}$, which is $2 \zeta_{r}=3$ in our case, and the fact that $r^{2}$ is a positive variable. So a $95 \%$ confidence interval is $m_{i} \pm \Delta r_{i}^{2}$ with $\Delta r_{i}^{2}=\chi \sigma_{r_{i, j}^{2}} k^{-1 / 2}$ for some real number $\chi$. Monte-Carlo simulations show $\chi=3.23$. Thus we can use the standard procedure for confidence intervals, with a factor $\chi=3.23$ rather than the Gaussian $\chi=1.95$.

\subsection{Test of the linear relation $E\left[r^{2} \mid Q\right]=\alpha+\beta V$}

For each bin $Q_{i}$ of $Q$, we set $r_{i}^{2}=E\left[r^{2} \mid Q=Q_{i}\right]$, and $\Delta r_{i}^{2}$ equal to the half-width of its $95 \%$ confidence interval $\left[r_{i}^{2}-\Delta r_{i}^{2}, r_{i}^{2}+\Delta r_{i}^{2}\right]$. By weighted least squares, with weights $\left(\Delta r_{i}^{2}\right)^{-2}$, we fit an affine relationship $E\left[r^{2} \mid Q\right]=g(Q)$, with

$$
\begin{gathered}
g(Q)=0.90+0.31 V \\
(0.057) \quad(0.013) \\
R^{2}=0.98
\end{gathered}
$$

The standard errors are in parentheses. We find that for all values $Q_{i} \geq 3$, the predicted value $g\left(Q_{i}\right)$ belongs to the $95 \%$ confidence interval:

$$
g\left(Q_{i}\right) \in\left[r_{i}^{2}-\Delta r_{i}^{2}, r_{i}^{2}+\Delta r_{i}^{2}\right] .
$$

\footnotetext{
${ }^{39}$ So we took the first moment for $V, r^{2}$ and $V / N$, and the second moment for $N, N^{\prime}, r$ and $N^{\prime} / N$.
} 
We conclude that, at the $95 \%$ confidence level, we cannot reject the linear form $E\left[r^{2} \mid Q\right]=g(Q)$ for $Q$ large enough, here $Q \geq 3$.

\section{References}

[1] Ané, Thierry and Hélyette Geman, "Order Flow, Transaction Clock, and Normality of Asset Returns," Journal of Finance, 55 (2000), 2259-84.

[2] Andersen, Torben G., Tim Bollerslev, Francis Diebold, and Heiko Ebens, "The Distribution of Realized Stock Return Volatility," Journal of Financial Economics, 61(2001), 43-76.

[3] Axtell, Robert "Zipf Distribution of U.S. Firm Sizes," Science, 293 (2001), 1818-20.

[4] Bak, Per, Ken Chen, José Scheinkman and Michael Woodford "Aggregate Fluctuations from Independent Sectoral Shocks: Self-Organized Criticality in a Model of Production and Inventory Dynamics," Ricerche Economiche, 47 (1993), 3-30.

[5] Barberis, Nicholas and Richard Thaler "A Survey of Behavioral Finance", in Handbook of the Economics of Finance, eds. George Constantinides, and René Stulz (North-Holland: Amsterdam, 2003).

[6] Biais, Bruno, Lawrence Glosten and Chester Spatt, "The Microstructure of Stock Markets," mimeo (2002).

[7] Bouchaud, Jean-Philippe and Marc Potters, Theory of Financial Risks: From Statistical Physics to Risk Management (New York : Cambridge University Press, 2000).

[8] Breen, William, Laurie Hodrick and Robert Korajczyk, "Predicting Equity Liquidity," Management Science 48 (2002) 470-483.

[9] Breiman, Leonard "On some limit theorems similar to the arc-sin law", Theory of probability and its applications, 10 (1965) 323-30.

[10] Burdett, Kenneth and Maureen O'Hara, "Building Blocks: An Introduction to Block Trading," Journal of Banking ES Finance, 11 (1987), 193-212.

[11] Campell, John and Robert Shiller "The Dividend-Price Ratio and Expectations of Future Dividends and Discount Factors", Review of Financial Studies 1 (1998), 195-228.

[12] Canning, David, Luis Amaral, Y. Lee, Martin Meyer, and H. Eugene Stanley, "Scaling in the Volatility of GDP Growth Rates," Economics Letters, 60 (1998), 335-341.

[13] Carhart, Mark, "On Persistence in Mutual Funds Performance," Journal of Finance, 52 (1997), $57-82$.

[14] Chan, Louis and Josef Lakonishok, "Institutional Trades and Intraday Stock Price Behavior," Journal of Financial Economics, 33 (1993), 173-189.

[15] Chan, Louis and Josef Lakonishok, "The Behavior of Stock Prices Around Institutional Trades," The Journal of Finance, 50 (1995), 1147- 1174.

[16] Corsetti, Giancarlo, Paolo Pesenti and Nouriel Roubini, The Role of Large Players in Currency Crises, in: S.Edwards and J. Frankel (eds.) Currency Crises in Emerging Markets: Crisis Prevention, NBER and Chicago University Press, (2002). 
[17] Coyne, Kevin and Jonathan Witter, "Taking the Mystery Out of Investor Behavior" Harvard Business Review (2002).

[18] Clark, Peter, "A Subordinated Stochastic Process Model with Finite Variance for Speculative Prices," Econometrica, 41 (1973), 135-155.

[19] Cutler, David, James Poterba and Lawrence Summers "What moves stock prices?" Journal of Portfolio Management 15, (1989) 4-12.

[20] Daniel, Kent, Daniel Hirshleifer and Avanidhar Subrahmanyam, "Investor Psychology and Security Market Under- and Over-reactions," Journal of Finance, 53 (1998), 1839-1885.

[21] Duffie, Darrell, Nicolae Garleanu and Lasse Pedersen, "Securities Lending, Shorting, and Pricing," Journal of Financial Economics, 66 (2002), 307-339.

[22] Easley, David, Soeren Hvidjkaer and Maureen O'Hara, "Is Information Risk a Determinant of Asset Prices?", forth. Journal of Finance (2003).

[23] Edelen, Roger and Jerold Warner, "Aggregate Price Effects of Institutional Trading: A Study of Mutual Fund Flow and Market Return," Journal of Financial Economics, 59 (2001), 195-220.

[24] Embrechts, Paul, Claudia Kluppelberg and Thomas Mikosch, Modelling Extremal Events (Springer Verlag, New York, 1997).

[25] Evans, Martin and Richard Lyons, "Order Flow and Exchange Rate Dynamics", Journal of Political Economy, 110 (2002), 170-180.

[26] Fair, Ray, "Events that shook the market", Journal of Business, 75 (2002), 713-731.

[27] Fama, Eugene, "Mandelbrot and the Stable Paretian Hypothesis" Journal of Business, 36 (1963), 420-429.

[28] Feenberg, Daniel and James Poterba, "Income Inequality and the Incomes of Very High-Income Taxpayers: Evidence from Tax Returns," in Tax Policy and the Economy, ed. James Poterba (Cambridge, MA: MIT Press, 1993), 145-77.

[29] French, Kenneth and Richard Roll, "Stock Return Variance: The Arrival of Information and the Reaction of Traders" Journal of Financial Economics 17 (1986), 99-117.

[30] Gabaix, Xavier, "Zipf's Law for Cities: An Explanation," Quarterly Journal of Economics 114 (1999), 739-67.

[31] Gabaix, Xavier, "Power Laws and the Origins of the Business Cycle," mimeo (2003).

[32] Gabaix, Xavier, Parameswaran Gopikrishnan, Vasiliki Plerou and H. Eugene Stanley, "A theory of power law distributions in financial market fluctuations," Nature, 423 (2003a), 267-230.

[33] Gabaix, Xavier, Parameswaran Gopikrishnan and Vasiliki Plerou, and H. Eugene Stanley, "Technical appendix" for the present paper, mimeo (2003b).

[34] Gabaix, Xavier, Parameswaran Gopikrishnan and Vasiliki Plerou, and H. Eugene Stanley, "Tobin tax and large fluctuations in financial activity", mimeo (2003c).

[35] Gabaix, Xavier, Parameswaran Gopikrishnan and Vasiliki Plerou, H. Eugene Stanley, "Are stock market crashes outliers?", mimeo (2003d) 
[36] Gabaix, Xavier, Rita Ramalho and Jonathan Reuter (2003) "Power laws and mutual fund dynamics", MIT mimeo (2003).

[37] Gabaix, Xavier and Yannis Ioannides, The evolution of the city size distribution, in Handbook of Urban and Regional Economics, 4 (2004) V. Henderson and J. Thisse ed., North Holland.

[38] Gnedenko, B. V., and A. N. Kolmogorov. Limit Distributions for Sums of Independent Random Variables (Addison-Wesley, Reading, MA. 1968).

[39] Gopikrishnan, Parameswaran, Martin Meyer, Luis Amaral and H. Eugene Stanley "Inverse Cubic Law for the Distribution of Stock Price Variations," European Physical Journal B, 3 (1998), 139-140.

[40] Gopikrishnan, Parameswaran, Vasiliki Plerou, Luis Amaral, Martin Meyer and H. Eugene Stanley "Scaling of the Distribution of Fluctuations of Financial Market Indices," Physical Review E, 60 (1999), 5305-5316.

[41] Gopikrishnan, Parameswaran, Vasiliki Plerou, Xavier Gabaix and H. Eugene Stanley "Statistical Properties of Share Volume Traded in Financial Markets," Physical Review E, 62 (2000), R4493-R4496.

[42] Grossman, Sanford and Merton Miller "Liquidity and Market Structure," Journal of Finance, 43 (1988), 617-33.

[43] Guillaume, Dominique M., Michel M. Dacorogna, Rakhal R. Davé, Ulrich A. Müller, Richard B. Olsen and Olivier V. Pictet, "From the Bird's Eye to the Microscope: a Survey of New Stylized Facts of the Intra-Daily Foreign Exchange Markets," Finance and Stochastics, 1 (1997), 95-129.

[44] Hasbrouck, Joel, "Trades, Quotes, Inventories, and Information," Journal of Financial Economics, 22 (1988), 229-252.

[45] Hasbrouck, Joel "Measuring the Information-Content of Stock Trades," Journal of Finance, 46 (1991), 179-207.

[46] Hasbrouck, Joel and Duane Seppi, "Common Factors in Rpices, Order Flows and Liquidity", Journal of Financial Economics, 59 (2001), 388-411.

[47] He, Hau and Jiang Wang, "Differential Information and Dynamic Behavior of Stock Trading Volume," Review of Financial Studies, 8 (1995), 919-972.

[48] Hirshleifer, David, "Investor psychology and asset pricing," Journal of Finance, 56 (2001), 1533-1598.

[49] Holthausen, Robert, Richard Leftwich and David Mayers "Large-Block Transactions, the Speed of Response, and Temporary and Permanent Stock-Price Effects," Journal of Financial Economics, 26 (1990), 71-95.

[50] Keim, Donald and Ananth Madhavan "The Upstairs Market for Large-Block Trades: Analysis and Measurement of Price Effects," Review of Financial Studies, 9 (1996), 1-36.

[51] Keim, Donald and Ananth Madhavan "Transaction Costs and Investment Style: An InterExchange Analysis of Institutional Equity Trades," Journal of Financial Economics, 46 (1997), 265-292. 
[52] Koski, Jennifer and Roni Michaely, "Prices, Liquidity, and the Information Content of Trades," Review of Financial Studies, 13 (2000), 659-696.

[53] Kyle, Albert "Continuous Auctions and Insider Trading," Econometrica, 53 (1985), 1315-1335.

[54] Lee, Charles and Mark Ready, "Inferring Trade Direction from Intraday Data," Journal of Finance, 46 (1991), 733-746.

[55] Lévy, Moshe, Heim Lévy and Sorin Solomon, Microscopic Simulation of Financial Markets: From Investor Behavior to Market Phenomena (San Diego: Academic Press, 2000).

[56] Liu, Yanhui, Parameswaran Gopikrishnan, Pierre Cizeau, Martin Meyer, Chung-Kang Peng and H. Eugene Stanley "Statistical properties of the volatility of price fluctuations," Physical Review E, 60 (1999), 1390-1400.

[57] Lillo, Fabrizio, Doyne Farmer and Rosario Mantegna, "Master curve for price-impact function", Nature, 421, (2003) 129-130.

[58] Lo, Andrew, Craig MacKinlay and June Zhang "Econometric models of limit-order executions," Journal of Financial Economics, 65 (2002), 31-72.

[59] Lo, Andrew and Jiang Wang, "Trading Volume: Definitions, Data Analysis, and Implications of Portfolio Theory", Review of Financial Studies, 13 (2001), 257-300.

[60] Logan, B.F., C. Mallows, S. Rice and L. Shepp "Limit distributions of self-normalized sums," Annals of Probability, 1 (1973), 788-809.

[61] Lux, Thomas, "The Stable Paretian Hypothesis and the Frequency of Large Returns: An Examination of Major German Stocks," Applied Financial Economics, 6 (1996), 463 - 475

[62] Lux, Thomas and Didier Sornette "On rational bubbles and fat tails," Journal of Money Credit and Banking, 34 (2002), 589-610.

[63] Madhavan, Ananth and Minder Cheng, "In Search of Liquidity: Block Trades in the Upstairs and Downstairs Markets," Review of Financial Studies, 10 (1997), 175-203.

[64] Madhavan, Ananth, Matthew Richardson and Mark Roomans "Why do Security Prices Fluctuate? A Transaction-Level Analysis of NYSE Stocks," Review of Financial Studies, 10 (1997), 1035-1064.

[65] Mandelbrot, Benoit, "The Variation of Certain Speculative Prices," Journal of Business, 36 (1963), 394-419.

[66] Mantegna, Rosario and H. Eugene Stanley, "Scaling Behavior in the Dynamics of an Economic Index," Nature, 376 (1995), 46-49.

[67] Mantegna, Rosario and H. Eugene Stanley, An Introduction to Econophysics: Correlations and Complexity in Finance(Cambridge: Cambridge University, 2000).

[68] Maslov, Sergei and Mark Mills, "Price Fluctuations from the Order Book Perspective: Empirical Facts and a Simple Model," Physica A, 299 (2001), 234-246.

[69] O’Hara, Maureen, Market Microstructure Theory (Cambridge, MA: Blackwell, 1995).

[70] Okuyama, K., M. Takayasu and Hideki Takayasu, "Zipf's Law in Income Distribution of Companies," Physica A, 269 (1999), 125-131 
[71] Piketty, Thomas and Emmanuel Saez, "Income Inequality in the United States, 1913-1998", Quarterly Journal of Economics, 118, (2003), 1-39.

[72] Plerou, Vasiliki, Parameswaran Gopikrishnan, Luis Amaral, Martin Meyer and H. Eugene Stanley "Scaling of the Distribution of Fluctuations of Financial Market Indices," Physical Review E, 60 (1999), 6519-6529.

[73] Plerou, Vasiliki, Parameswaran Gopikrishnan, Luis Amaral, Xavier Gabaix and H. Eugene Stanley "Economic Fluctuations and Anomalous Diffusion," Physical Review E, 62 (2000), R3023R3026.

[74] Plerou, Vasiliki, Parameswaran Gopikrishnan, Xavier Gabaix, Luis Amaral and H. Eugene Stanley, "Price Fluctuations, Market Activity, and Trading Volume," Quantitative Finance, 1 (2001), 262-269.

[75] Plerou, Vasiliki, Parameswaran Gopikrishnan, Xavier Gabaix and H. Eugene Stanley, "Quantifying Stock Price Response to Demand Fluctuations", Physical Review E, 66 (2002) 027104.

[76] Plerou, Vasiliki, Parameswaran Gopikrishnan, Xavier Gabaix and H. Eugene Stanley, "Power laws in finance: Evidence from the Paris Bourse", mimeo (2003).

[77] Roll, Richard, " $R^{2}$ ", Journal of Finance, 43 (1988), 541-566.

[78] Saar, Gideon, "Price Impact Asymmetry of Block Trades: An Institutional Trading Explanation," Review of Financial Studies,14 (2001), 1153-81.

[79] Shiller, Robert, Market Volatility, (Cambridge, MA: MIT, 1989).

[80] Shleifer, Andrei "Do Demand Curves for Stocks Slope Down?" Journal of Finance 41 (1986), $579-90$.

[81] Shleifer, Andrei, Inefficient Markets: an Introduction to Behavioral Finance (Oxford University, 2000).

[82] Shleifer, Andrei and Robert Vishny "The Limits of Arbitrage," Journal of Finance, 52 (1997), $35-55$.

[83] Solomon, Solomon and Peter Richmond "Power Laws of Wealth, Market Order Volumes and Market Returns," Physica A, 299 (2001), 188-197.

[84] Sornette, Didier, Critical Phenomena in Natural Sciences : Chaos, Fractals, Self-organization and Disorder : Concepts and Tools, (Heidelberg and New York: Springer 2000).

[85] Stoll, Hans, "The supply of dealer services in securities markets" Journal of Finance 33, (1978) $1133-1151$.

[86] United States' Presidential Task Force on Market Mechanisms, Report of the Presidential Task Force on Market Mechanisms submitted to the President of the United States. (Washington, D.C. Docs., U.S. G.P.O., 1988).

[87] Vayanos, Dimitri "Strategic Trading in a Dynamic Noisy Market," Journal of Finance, 55 (2001), 131-171.

[88] Wang, Jiang, "A model of competitive stock trading volume", Journal of Political Economy, 102 (1994), 127-168.

[89] Zhang, Yi-Cheng. "Toward a theory of marginally efficient markets", Physica A, 269 (1999). 